\title{
Isomonodromy sets of accessory parameters for Heun class equations
}

\author{
Jun Xia*, Shuai-Xia Xu ${ }^{\dagger}$ and Yu-Qiu Zhao*
}

\begin{abstract}
In this paper, we consider the monodromy and, in particularly, the isomonodromy sets of accessory parameters for the Heun class equations. We show that the Heun class equations can be obtained as limits of the linear systems associated with the Painlevé equations when the Painlevé transcendents go to one of the actual singular points of the linear systems. While the isomonodromy sets of accessory parameters for the Heun class equations are described by the Taylor or Laurent coefficients of the corresponding Painlevé functions, or the associated tau functions, at the positions of the critical values. As an application of these results, we derive some asymptotic approximations for the isomonodromy sets of accessory parameters in the Heun class equations, including the confluent Heun equation, the doubly-confluent Heun equation and the reduced biconfluent Heun equation.
\end{abstract}

2010 mathematics subject classification: 33E17; 34A30; 34E05; 34M55; 41A60

Keywords and phrases: Heun class equations, Painlevé equations, accessory parameter, monodromy, isomonodromy deformation, asymptotic analysis.

\section{Contents}

1 Introduction and statement of results

2 Accessory parameters of RTHE

2.1 Monodromy of RTHE . . . . . . . . . . . . . . . . . .

2.2 Isomonodromy deformation and PI equation . . . . . . . . . . . .

2.3 Reduction of the linear system for PI to RTHE . . . . . . . . . . . . .

2.4 Isomonodromy set of accessory parameters of RTHE

3 Accessory parameters of THE

3.1 Monodromy of THE . . . . . . . . . . . . . . . . . . . 9

3.2 Isomonodromy deformation and PII equation . . . . . . . . . . . . . . 9

3.3 Reduction of the linear system for PII to THE . . . . . . . . . . . . . . 11

3.4 Isomonodromy set of accessory parameters of THE . . . . . . . . . . . . . 12

4 Accessory parameters of RBHE 12

4.1 Monodromy of RBHE . . . . . . . . . . . . . . . . . . 12

4.2 Isomonodromy deformation and PXXXIV equation . . . . . . . . . . . . 13

4.3 Reduction of the linear system for PXXXIV to RBHE . . . . . . . . . . . . 14

4.4 Isomonodromy set of accessory parameters of RBHE . . . . . . . . . . . . 15

\footnotetext{
*Department of Mathematics, Sun Yat-sen University, GuangZhou 510275, China.

†Institut Franco-Chinois de l'Energie Nucléaire, Sun Yat-sen University, GuangZhou 510275, China.
} 
5 Accessory parameters of DHE 15

5.1 Monodromy of DHE . . . . . . . . . . . . . . . . . 15

5.2 Isomonodromy deformation and PIII equation . . . . . . . . . . . . . 16

5.3 Reduction of the linear system for PIII to DHE . . . . . . . . . . . . 18

5.4 Isomonodromy set of accessory parameters of DHE . . . . . . . . . . . . 19

6 Accessory parameters of BHE $\quad 19$

6.1 Monodromy of BHE . . . . . . . . . . . . . . . . . . 19

6.2 Isomonodromy deformation and PIV equation . . . . . . . . . . . . . . 20

6.3 Reduction of the linear system for PIV to BHE . . . . . . . . . . . . . . 22

6.4 Isomonodromy set of accessory parameters of BHE . . . . . . . . . . . . . 23

7 Accessory parameters of CHE 24

7.1 Monodromy of CHE . . . . . . . . . . . . . . . . 24

7.2 Isomonodromy deformation and $\mathrm{PV}$ equation $\ldots \ldots \ldots \ldots \ldots . \ldots \ldots$

7.3 Reduction of the linear systems for PV to CHE . . . . . . . . . . . . . . 26

7.4 Isomonodromy set of accessory parameters of CHE . . . . . . . . . . . 28

8 Accessory parameters of $\mathrm{HE} \quad 29$

8.1 Reduction of the linear system for PVI to HE . . . . . . . . . . . . 29

8.2 Isomonodromy set of accessory parameters of HE . . . . . . . . . . . . . 35

9 Asymptotics of the accessory parameters 36

9.1 Asymptotics of the accessory parameter of RBHE . . . . . . . . . . . . 37

9.2 Asymptotics of the accessory parameter of CHE . . . . . . . . . . . . 38

9.3 Asymptotics of the accessory parameter of DHE . . . . . . . . . . . . . 39

\section{Introduction and statement of results}

The Heun equation (HE) is the general second-order linear ODE having four regular singular points, with the canonical form [25, Eq. (31.2.1)]

$$
\frac{\mathrm{d}^{2} w}{\mathrm{~d} z^{2}}+\left(\frac{\gamma}{z}+\frac{\delta}{z-1}+\frac{\epsilon}{z-a}\right) \frac{\mathrm{d} w}{\mathrm{~d} z}+\frac{\alpha \beta z-q}{z(z-1)(z-a)} w=0,
$$

where $\alpha+\beta+1=\gamma+\delta+\epsilon$. The parameters $\alpha, \beta, \gamma, \delta, \epsilon$ determine the characteristic exponents of the regular singularities at $z=0,1, a, \infty$ : the exponents are $\{0,1-\gamma\},\{0,1-\delta\},\{0,1-\epsilon\}$ and $\{\alpha, \beta\}$, respectively. While the remaining parameters $a$ and $q$, known as the accessory parameters, involve global monodromy properties of (1.1).

The same as the classical Gauss hypergeometric equation, the Heun equation has several confluent forms. Indeed, there are four standard confluent forms when two or more singularities merge into one or more irregular singularities (cf. [25, Eqs. (31.12.1)-(31.12.4)]).

(i) Confluent (or, singly-confluent) Heun equation (CHE):

$$
\frac{\mathrm{d}^{2} w}{\mathrm{~d} z^{2}}+\left(1+\frac{\gamma}{z}+\frac{\delta}{z-a}\right) \frac{\mathrm{d} w}{\mathrm{~d} z}+\frac{p z-q}{z(z-a)} w=0 .
$$

This equation has two regular singularities, and an irregular singularity of rank 1 at infinity arising from the coalescing of two regular singularities.

(ii) Doubly-confluent Heun equation (DHE):

$$
\frac{\mathrm{d}^{2} w}{\mathrm{~d} z^{2}}+\left(\frac{1}{2}+\frac{\gamma}{z}-\frac{a^{2}}{2 z^{2}}\right) \frac{\mathrm{d} w}{\mathrm{~d} z}+\frac{p z-q}{z^{2}} w=0 .
$$


This equation has two irregular singularities of rank 1 at zero and infinity, each originating from the confluence of two regular singularities.

(iii) Biconfluent Heun equation (BHE):

$$
\frac{\mathrm{d}^{2} w}{\mathrm{~d} z^{2}}+\left(2 z+2 a+\frac{\gamma}{z}\right) \frac{\mathrm{d} w}{\mathrm{~d} z}+\frac{p z-q}{z} w=0 .
$$

This equation possesses a regular singularity, and an irregular singularity of rank 2 at infinity arising from the coalescing of three regular singularities.

(iv) Triconfluent Heun equation (THE):

$$
\frac{\mathrm{d}^{2} w}{\mathrm{~d} z^{2}}+\left(2 z^{2}+a\right) \frac{\mathrm{d} w}{\mathrm{~d} z}+(p z-q) w=0 .
$$

This equation has one irregular singularity of rank 3 at infinity, resulting from the coalescing of all of the four singularities.

Modified or reduced forms of the confluent Heun equations are also available. Five reduced confluent equations appear as a result of weak confluence processes. For instance, we have the reduced triconfluent Heun equation (RTHE)

$$
\frac{\mathrm{d}^{2} w}{\mathrm{~d} z^{2}}-\left(4 z^{3}+2 a z+q\right) w=0,
$$

which has one irregular singularity of rank $5 / 2$ at infinity. There is the reduced biconfluent Heun equation (RBHE)

$$
\frac{\mathrm{d}^{2} w}{\mathrm{~d} z^{2}}+\frac{2 \alpha}{z} \frac{\mathrm{d} w}{\mathrm{~d} z}-\left(z+a+\frac{q}{z}\right) w=0
$$

with one irregular singularity of rank $3 / 2$ at infinity and one regular singularity at the origin. Also, ten more equations are to be added to the list, if elementary singularities are regarded as special types of singularities; see [31].

The HE, together with all of its confluent forms, is called the Heun class of equations; see [29]. This class of equations has numerous applications in theory of black holes, general relativity, polymer and chemical physics, astrophysics, molecular physics, crystalline materials and cosmology, etc.; see [17, 25, 26, 31 and the references therein.

Painlevé equations and Painlevé transcendents will be the main tools in the present investigation. By Painlevé equations we mean six equations denoted by PI-PVI. The six Painlevé equations were first introduced by Paul Painlevé and his coworkers at the turn of the twentieth century in the classification of second-order ODEs of the form

$$
y_{x x}=F\left(x, y, y_{x}\right)
$$

with $F$ meromorphic in $x$ and rational in $y$ and $y_{x}$, whose solutions have the Painlevé property, that is, the only movable singularities of their solutions are poles or isolated essential singularities; see [16, 25]. The Painlevé equations find important applications in various fields, such as number theory, statistical mechanics, random matrix theory, orthogonal polynomials, quantum gravity and quantum field theory, nonlinear optics and fibre optics, etc.; see [5, 12, 13, 25] and the references therein.

In [14, 15, Fuchs discovered a remarkable connection between the HE and the PVI. He added an extra apparent singularity at $z=y$ to the HE (1.1). The apparent singularity is presented in the equation but is absent in the solution. The position of the apparent singularity $y$ is deformed with the regular singularity $a$ in (1.1). Then, the PVI equation arose as a compatibility condition, of the deformed HE coupled with another linear equation with differentiation in the 
variable $a$. Inspired by the works of Fuchs, Slavyanov et al. studied the deformation of the Heun class of equations by adding an apparent singularity. It was shown that each Painlevé equation can be considered as the isomonodromy deformation condition for a deformed equation of the Heun class; see [30]-32. In [29, Slavyanov also discovered that the Heun class equations can be regarded as the quantization of the classical Hamiltonian of the Painlevé equations. In this sense, there exists the following correspondence between Heun class equations and Painlevé equations:

$$
\mathrm{HE} \rightarrow \mathrm{PVI}, \mathrm{CHE} \rightarrow \mathrm{PV}, \mathrm{BHE} \rightarrow \mathrm{PIV}, \mathrm{DHE} \rightarrow \mathrm{PIII}, \mathrm{THE} \rightarrow \mathrm{PII}, \mathrm{RTHE} \rightarrow \mathrm{PI} .
$$

Here ' $\rightarrow$ ' means that given an equation of Heun class there is a Painlevé equation corresponding to it.

It is well-known that every Painlevé equation can be obtained as the compatibility condition of a $2 \times 2$ matrix linear system (also called Lax pair) [20]:

$$
\frac{\partial \Phi(z, x)}{\partial z}=A(z, x) \Phi(z, x)
$$

and

$$
\frac{\partial \Phi(z, x)}{\partial x}=B(z, x) \Phi(z, x) .
$$

The first-order matrix equation (1.9) is equivalent to two second-order linear ODEs satisfied respectively by the elements of the first row and second row of $\Phi(z, x)$. More precisely, if we denote the coefficient matrix $A(z, x)$ by

$$
A(z, x):=A=\left(\begin{array}{ll}
A_{11} & A_{12} \\
A_{21} & A_{22}
\end{array}\right),
$$

then each element of the first row of $\Phi(z, x)$ satisfies the ODE:

$$
\frac{\mathrm{d}^{2} \Phi_{1}}{\mathrm{~d} z^{2}}-\left(\operatorname{Tr} A+\frac{A_{12}^{\prime}}{A_{12}}\right) \frac{\mathrm{d} \Phi_{1}}{\mathrm{~d} z}+\left(\operatorname{det} A-A_{11}^{\prime}+A_{11} \frac{A_{12}^{\prime}}{A_{12}}\right) \Phi_{1}=0,
$$

while the elements of the second row solve a similar ODE:

$$
\frac{\mathrm{d}^{2} \Phi_{2}}{\mathrm{~d} z^{2}}-\left(\operatorname{Tr} A+\frac{A_{21}^{\prime}}{A_{21}}\right) \frac{\mathrm{d} \Phi_{2}}{\mathrm{~d} z}+\left(\operatorname{det} A-A_{22}^{\prime}+A_{22} \frac{A_{21}^{\prime}}{A_{21}}\right) \Phi_{2}=0 .
$$

Here, the prime indicates the differentiation with respect to $z$.

It was observed in [12, p.86] that the linear equation (1.11) for PVI is equivalent to HE when the solutions to PVI approach the critical values $0,1, x, \infty$, which are the actual singular points of (1.11). Recently, Dubrovin and Kapaev [10] studied in detail the equivalence of HE and the Lax pair for PVI at the movable poles of the solutions of PVI. Moreover, they established a connection between the accessory parameter of $\mathrm{HE}$ and the free parameter of the Laurent expansion of PVI. Similar results were also derived earlier in [22]. In [6, 7], the equivalence of CHE and the Lax pair for PV at certain critical value of PV was shown and the accessory parameter was expressed in terms of the $\tau$-function of PV. An application to black holes was also addressed therein.

In this paper, we consider the monodromy and isomonodromy deformation of the Heun class equations. Firstly, we describe the monodromy of Heun class equations and consider the isomonodromy deformation by using the linear systems for the corresponding Painlevé equations. We show that the Heun class equations can be obtained as limits of the linear system 
(1.11) or (1.12) associated with the Painlevé equations I-VI and XXXIV as the corresponding Painlevé transcendents $y(x)$ approach one of the actual singular points of the linear system:

$$
\mathrm{PI} \rightarrow \mathrm{RTHE}, \quad \mathrm{PII} \rightarrow \mathrm{THE}, \quad \mathrm{PXXXIV} \rightarrow \mathrm{RBHE}, \quad \mathrm{PIII} \rightarrow \mathrm{DHE},
$$

and

$$
\mathrm{PIV} \rightarrow \mathrm{BHE}, \quad \mathrm{PV} \rightarrow \mathrm{CHE}, \quad \mathrm{PVI} \rightarrow \mathrm{HE} .
$$

Moreover, the accessory parameters in these equations of Heun class are determined by the corresponding Painlevé functions and $\tau$-functions. Secondly, using the the limiting procedure in (1.13), (1.14) and the monodromy of a specific Heun class equation, we show that there is a discrete set of pairs of accessory parameters $\left(a_{n}, q_{n}\right)$, such that the equation of Heun class corresponding to these parameters has the same monodromy data. Under a bijection, the discrete set coincides with the set of parameters $\left(a_{n}, b_{n}\right)$ in the Taylor or Laurent expansions, respectively at the zeros or poles $a_{n}$, of the unique solution to the corresponding Painlevé equation with the same monodromy data as the Heun class equation. Finally, using known asymptotic expansions for the Painlevé transcendents and the associated $\tau$-functions in the literature, we derive some asymptotic approximations for the isomonodromy sets of accessory parameters in the Heun class equations.

The rest of this paper is organized in the following way. We consider the monodromy data and isomonodromy deformation of the Heun class equations RTHE-HE in Sections $2-$ 8. respectively. We describe the isomonodromy sets of accessary parameters by the sets of parameters in the Taylor or Laurent expansion near the zeros or poles of the solution of the corresponding Painlevé equation with the same monodromy data as the Heun class equation. The main results are stated in Theorems 1 7 at the end of each section. The proofs of Theorem 1 and Theorem 7 are given in Section 2 and Section 8, respectively, with details included. These proofs are concerned with two representative examples of Heun class equations, namely the HE with four regular singularities and the RTHE with an irregular singularity. While Theorems 2 6 can be proved in the same manner and we skip the detail. In the last section, we derive asymptotic approximations for isomonodromy sets of accessory parameters of some Heun class equations, expressed in terms of the monodromy data. The equations of Heun class we considered in this section include the RBHE, CHE, and DHE.

\section{Accessory parameters of RTHE}

\subsection{Monodromy of RTHE}

Consider the RTHE equation (1.6) with parameters $a$ and $q$. There exist unique solutions, of the form $Y_{k}(z)=\left(y_{k 1}(z), y_{k 2}(z)\right)$, which satisfy the normalized asymptotic behavior as $z \rightarrow \infty$

$$
Y_{k}(z) \sim z^{-\frac{3}{4}}(1,1) e^{\left(\frac{4}{5} z^{\frac{5}{2}}+a z^{\frac{1}{2}}\right) \sigma_{3}}, \quad z \in \Omega_{k},
$$

where $\Omega_{k}, k=-1, \cdots, 4$, are the Stokes sectors defined by

$$
\Omega_{k}=\left\{z \in \mathbb{C}: \frac{\pi}{5}(2 k-5)<\arg z<\frac{\pi}{5}(2 k-1)\right\},
$$

and $\sigma_{3}$ is one of the Pauli matrices,

$$
\sigma_{1}=\left(\begin{array}{cc}
0 & 1 \\
1 & 0
\end{array}\right), \quad \sigma_{2}=\left(\begin{array}{cc}
0 & -i \\
i & 0
\end{array}\right) \quad \text { and } \quad \sigma_{3}=\left(\begin{array}{cc}
1 & 0 \\
0 & -1
\end{array}\right)
$$


The solutions are related to each other by the Stokes matrices

$$
Y_{k+1}(z)=Y_{k}(z) S_{k}, \quad k=-1, \cdots, 3,
$$

where

$$
S_{2 k-1}=\left(\begin{array}{cc}
1 & 0 \\
s_{2 k-1} & 1
\end{array}\right), \quad S_{2 k}=\left(\begin{array}{cc}
1 & s_{2 k} \\
0 & 1
\end{array}\right) .
$$

Moreover, it follows from the asymptotic behavior in (2.1) that

$$
Y_{4}\left(e^{2 \pi i} z\right)=i Y_{-1}(z) \sigma_{1} .
$$

Using (2.3)-(2.5), we find the cyclic condition

$$
S_{-1} \cdots S_{3}=i \sigma_{1} .
$$

The restriction (2.6) contributes four scalar algebraic equations, three among them are independent. Accordingly, the Stokes multipliers $s_{k}$ fulfill

$$
s_{k}=i\left(1+s_{k+2} s_{k+3}\right)
$$

for all integer $k$, with $s_{k+5}=s_{k}$. The monodromy data is constituted by the set of Stokes multipliers, which is a 2 -dimensional surface in $\mathbb{C}^{5}$ described by (2.7)

$$
\left\{\left(s_{1}, s_{2}, s_{3}, s_{4}, s_{5}\right) \in \mathbb{C}^{5}: s_{1}, \ldots s_{5} \text { satisfy (2.7) }\right\} .
$$

\subsection{Isomonodromy deformation and PI equation}

To study the isomonodromy deformation of the RTHE equation, it is convenient to consider a $2 \times 2$ matrix system, which has one irregular singular point of rank $5 / 2$ at infinity. In [12, 20], the isomonodromy deformation of such a matrix system has been considered. We review it briefly below in this section.

Consider the following Lax pair for PI parameterized in the way as [20, (C.2)]

$$
\left\{\begin{array}{l}
\frac{\partial \Phi(z, x)}{\partial z}=A(z, x) \Phi(z, x) \\
\frac{\partial \Phi(z, x)}{\partial x}=B(z, x) \Phi(z, x)
\end{array}\right.
$$

where

$$
A(z, x)=\left(\begin{array}{cc}
-v & z^{2}+y z+y^{2}+\frac{x}{2} \\
4(z-y) & v
\end{array}\right), \quad B(z, x)=\left(\begin{array}{cc}
0 & \frac{z}{2}+y \\
2 & 0
\end{array}\right) .
$$

The compatibility condition of the Lax pair reads

$$
\left\{\begin{array}{l}
\frac{\mathrm{d} y}{\mathrm{~d} x}=v, \\
\frac{\mathrm{d} v}{\mathrm{~d} x}=6 y^{2}+x,
\end{array}\right.
$$

which leads to the PI equation

$$
\frac{\mathrm{d}^{2} y}{\mathrm{~d} x^{2}}=6 y^{2}+x
$$

The $\tau$-function associated with PI is defined by (see [20, (C.7)])

$$
\sigma(x)=\frac{\mathrm{d}}{\mathrm{d} x} \log \tau(x)=\frac{1}{2} v^{2}-2 y^{3}-x y .
$$


Then, we have (see [20, (C.9)])

$$
\left(\frac{\mathrm{d}^{2} \sigma}{\mathrm{d} x^{2}}\right)^{2}+4\left(\frac{\mathrm{d} \sigma}{\mathrm{d} x}\right)^{2}+2 x \frac{\mathrm{d} \sigma}{\mathrm{d} x}-2 \sigma=0 .
$$

There exist unique solutions $\Phi_{k}(z, x)$ of the equation (2.9), which satisfy the normalized asymptotic behavior as $z \rightarrow \infty$

$$
\Phi_{k}(z, x)=\left(\frac{z}{4}\right)^{\frac{1}{4} \sigma_{3}} \frac{\sigma_{3}+\sigma_{1}}{i \sqrt{2}}\left(I+O\left(z^{-\frac{1}{2}}\right)\right) e^{\left(\frac{4}{5} z^{\frac{5}{2}}+x z^{\frac{1}{2}}\right) \sigma_{3}}, \quad z \in \Omega_{k},
$$

where $\Omega_{k}$ for $k=-1, \cdots, 4$ are the Stokes sectors defined by (2.2). From (2.13), we see that the solutions are related to each other by the Stokes matrices

$$
\Phi_{k+1}(z)=\Phi_{k}(z) \hat{S}_{k}, \quad k=-1, \cdots, 3,
$$

where

$$
\hat{S}_{2 k-1}=\left(\begin{array}{cc}
1 & 0 \\
\hat{s}_{2 k-1} & 1
\end{array}\right), \quad \hat{S}_{2 k}=\left(\begin{array}{cc}
1 & \hat{s}_{2 k} \\
0 & 1
\end{array}\right) .
$$

The behavior (2.13) also gives

$$
\Phi_{4}\left(e^{2 \pi i} z\right)=i \Phi_{-1}(z) \sigma_{1} .
$$

From (2.14)-(2.16), we find the cyclic condition

$$
\hat{S}_{-1} \hat{S}_{0} \cdots \hat{S}_{3}=i \sigma_{1} .
$$

This condition implies the following algebraic equations

$$
\hat{s}_{k}=i\left(1+\hat{s}_{k+2} \hat{s}_{k+3}\right),
$$

with $k \in \mathbb{Z}$ and $\hat{s}_{k+5}=\hat{s}_{k}$. Thus, the Stokes multipliers are described by the 2-dimensional surface (2.8) in $\mathbb{C}^{5}$ with the coordinates $\left(\hat{s}_{k}\right)_{1 \leq k \leq 5}$.

\subsection{Reduction of the linear system for PI to RTHE}

In this subsection, we show that the RBHE can be obtained as a limit of the linear system (1.12) associated with the Lax pair for the Painlevé I equation when $x$ tends to one of the poles of the Painlevé I transcendents.

Substituting the enties of $A(z, x)$ into (1.12), we obtain

$$
\frac{\mathrm{d}^{2} \Phi_{2}}{\mathrm{~d} z^{2}}-\frac{1}{z-y} \frac{\mathrm{d} \Phi_{2}}{\mathrm{~d} z}-Q(z, x) \Phi_{2}=0,
$$

where

$$
\begin{aligned}
Q(z, x) & =4 z^{3}+2 x z+v^{2}-4 y^{3}-2 x y-\frac{v}{z-y} \\
& =4 z^{3}+2 x z+2 \frac{\mathrm{d}}{\mathrm{d} x} \log \tau(x)-\frac{v}{z-y} .
\end{aligned}
$$

To obtain RTHE, we need to eliminate the singularity $z=y$ in (2.19). This can be realized by considering the poles of $y$. It is known that $y$ admits the following Laurent expansion near a pole (see [16, Eq. (1.1)] ):

$$
y(x)=\frac{1}{(x-a)^{2}}-\frac{a}{10}(x-a)^{2}-\frac{1}{6}(x-a)^{3}+b(x-a)^{4}+O\left((x-a)^{5}\right),
$$


where $b$ is an arbitrary parameter.

Using the first equation of (2.10) to replace $v$ by $y^{\prime}$ and substituting (2.20) into the expression of $Q$ in (2.19), we obtain the RTHE as $x \rightarrow a$

$$
\frac{\mathrm{d}^{2} \Phi_{2}}{\mathrm{~d} z^{2}}-\left(4 z^{3}+2 a z+q\right) \Phi_{2}=0,
$$

with the accessory parameter $q$ given by

$$
q=\lim _{x \rightarrow a} 2\left(\frac{\mathrm{d}}{\mathrm{d} x} \log \tau(x)-\frac{1}{x-a}\right)=-28 b .
$$

\subsection{Isomonodromy set of accessory parameters of RTHE}

As shown in Section 2.3, the isomonodromy deformation of (2.9) is described by the PI equation. While the RTHE can be obtained as a limit of the second row of the isomonodromy family of $\Phi(z, x)$, when $x$ tends to one of the pole of the Painlevé I transcendent. The accessory parameters of RTHE are then expressed in terms of the parameters in the Laurent expansion near the poles of the PI transcendents. As a consequence, we obtain a family of accessory parameters such that the corresponding RTHE shares the same monodromy data, as stated in the following theorem.

Theorem 1. There is a discrete set of pairs of accessory parameters $\left(a_{n}, q_{n}\right)$ such that the RTHE (1.6) corresponding to these parameters has the same monodromy data (2.8) as the original one with the parameters $a$ and $q$. This set coincides, via the transformation $q_{n}=-28 b_{n}$, with the set of parameters $\left(a_{n}, b_{n}\right)$ in the Laurent expansion (2.20) near the poles $a_{n}$, of the unique solution of PI corresponding to the same monodromy data (2.8) as the RTHE.

Proof. Consider the RTHE (1.6) with given accessory parameters $a$ and $q$. There exist unique solutions of (1.6) which satisfy the normalized asymptotic behavior (2.1) as $z \rightarrow \infty$. The solutions are related by the Stokes matrices $S_{k}$ as given in (2.3) and (2.4), while the independent Stokes multipliers constitute the monodromy data (2.8) for the RTHE. Moreover, there exist unique solutions $\Phi_{k}(z, x)$ of the system (2.9) with the normalized behavior (2.13) at infinity, where the $x$-dependence of $\Phi_{k}(z, x)$ is described by the unique solution of the PI equation having the Laurent expansion (2.20). Here we let $a$ be a pole of the PI solution and choose the parameter $b$ according to (2.22), namely $b=-\frac{q}{28}$. The solutions $\Phi_{k}(z, x)$ are related by the Stokes matrices $\hat{S}_{k}$ defined in (2.14) and (2.15), which are independent of $x$ and have the same triangular-matrix forms as the Stokes matrices $S_{k}$ for the RTHE.

From the limiting procedure demonstrated in Section 2.3. we obtain the RTHE equation with accessory parameters $a$ and $q$, as a limit of the second row of the isomonodromy family $\Phi_{k}(z ; x)$ when $x \rightarrow a$. It follows from the $x$-independence of the monodromy data that the Stokes matrices $S_{k}=\hat{S}_{k}$. Thus, we have shown that any given accessory parameters $(a, q)$ is related, via $q=-28 b$, to the pole parameters $(a, b)$ of the unique solution of PI corresponding to the same monodromy data (2.8). It is known that each Painlevé tanscendents has infinity many poles which are discrete in the complex plane. Therefore the set of pairs of accessory parameters of RTHE sharing the same monodromy data are also discrete. Thus, we complete the proof of Theorem 1. 


\section{Accessory parameters of THE}

\subsection{Monodromy of THE}

Consider the THE (1.5) with the accessory parameters $a$ and $q$ and the fixed parameter $p$. There exist unique solutions of (1.5) with normalized asymptotic behavior as $z \rightarrow \infty$

$$
Y_{k}(z) \sim e^{-\left(\frac{1}{3} z^{3}+\frac{a}{2} z\right)} z^{-1}(1,1) e^{\left(\frac{1}{3} z^{3}+\frac{a}{2} z\right) \sigma_{3}} z^{\left(\mu-\frac{1}{2}\right) \sigma_{3}}, \quad z \in \Omega_{k},
$$

where the Stokes sectors are defined by

$$
\Omega_{k}=\left\{z \in \mathbb{C}: \frac{\pi}{6}(2 k-3)<\arg z<\frac{\pi}{6}(2 k+1)\right\},
$$

for $k=1, \cdots, 7$. Here the parameter $\mu$ is given by $\mu=(3-p) / 2$. The solutions are related by the Stokes matrices

$$
Y_{k+1}(z)=Y_{k}(z) S_{k}, \quad k=1, \ldots, 6,
$$

where

$$
S_{2 k-1}=\left(\begin{array}{cc}
1 & s_{2 k-1} \\
0 & 1
\end{array}\right), \quad S_{2 k}=\left(\begin{array}{cc}
1 & 0 \\
s_{2 k} & 1
\end{array}\right) .
$$

The behavior (3.1) also gives

$$
Y_{7}\left(e^{2 \pi i} z\right)=Y_{1}(z) e^{\pi i(2 \mu-1) \sigma_{3}} .
$$

We define the monodromy data of THE by

$$
\left\{S_{k}\right\}_{k=1}^{6} \text {. }
$$

From (3.3)-(3.5), we see that the Stokes matrices fulfill the cyclic condition

$$
S_{1} S_{2} \cdots S_{6}=e^{\pi i(2 \mu-1) \sigma_{3}} .
$$

Thus, the Stokes multipliers $s_{k}, k=1, \cdots, 6$ are constrained by the following algebraic equations

$$
\left\{\begin{array}{l}
1+s_{1} s_{2}+e^{2 \pi i \mu}\left(1+s_{4} s_{5}\right)=0 \\
1+s_{2} s_{3}+e^{-2 \pi i \mu}\left(1+s_{5} s_{6}\right)=0 \\
s_{1}+s_{3}+s_{1} s_{2} s_{3}-e^{2 \pi i \mu} s_{5}=0
\end{array}\right.
$$

These equations describe a 3 -dimensional surface in $\mathbb{C}^{6}$ with the coordinates $\left(s_{k}\right)_{1 \leq k \leq 6}$.

\subsection{Isomonodromy deformation and PII equation}

To study the isomonodromy deformation of THE, it is convenient to consider a $2 \times 2$ matrix system, which has one irregular singular point of rank 3 at infinity. In [20], the isomonodromy deformation of the matrix system with such an irregular singular point has been considered.

Recall the following Lax pair for PII with the parameterization given in [20, (C.10)-(C.11)]:

$$
\left\{\begin{array}{l}
\frac{\partial \Phi(z, x)}{\partial z}=A(z, x) \Phi(z, x) \\
\frac{\partial \Phi(z, x)}{\partial x}=B(z, x) \Phi(z, x)
\end{array}\right.
$$


where

$$
A(z, x)=\left(\begin{array}{cc}
z^{2}+v+\frac{x}{2} & u(z-y) \\
-\frac{2}{u}\left(v z+v y+\frac{1}{2}-\mu\right) & -z^{2}-v-\frac{x}{2}
\end{array}\right), \quad B(z, x)=\left(\begin{array}{cc}
\frac{1}{2} z & \frac{1}{2} u \\
-\frac{v}{u} & -\frac{1}{2} z
\end{array}\right) .
$$

The compatibility condition of the above Lax pair gives

$$
\left\{\begin{array}{l}
\frac{\mathrm{d} y}{\mathrm{~d} x}=y^{2}+v+\frac{x}{2} \\
\frac{\mathrm{d} v}{\mathrm{~d} x}=-2 y v-\frac{1}{2}+\mu \\
\frac{\mathrm{d} u}{\mathrm{~d} x}=-u y
\end{array}\right.
$$

which yields the PII equation

$$
\frac{\mathrm{d}^{2} y}{\mathrm{~d} x^{2}}=2 y^{3}+x y+\mu
$$

The PII $\tau$-function is defined by (see [20, (C.15)])

$$
\frac{\mathrm{d}}{\mathrm{d} x} \log \tau(x)=\frac{1}{2} v^{2}+\left(y^{2}+\frac{x}{2}\right) v+\left(\frac{1}{2}-\mu\right) y .
$$

There exist unique solutions $\Phi_{k}(z, x)$ of the equation (3.9) specified by the normalized asymptotic behavior as $z \rightarrow \infty$

$$
\Phi_{k}(z, x)=(I+O(1 / z)) e^{\left(\frac{1}{3} z^{3}+\frac{1}{2} x z\right) \sigma_{3}} z^{\left(\mu-\frac{1}{2}\right) \sigma_{3}}, \quad z \in \Omega_{k},
$$

where $\Omega_{k}$ for $k=1, \cdots, 7$ are the Stokes sectors defined in (3.2). From (3.13), we see that the solutions are related to each other by the Stokes matrices

$$
\Phi_{k+1}(z)=\Phi_{k}(z) \hat{S}_{k}, \quad k=1, \cdots, 6,
$$

where

$$
\hat{S}_{2 k-1}=\left(\begin{array}{cc}
1 & \hat{s}_{2 k-1} \\
0 & 1
\end{array}\right), \quad \hat{S}_{2 k}=\left(\begin{array}{cc}
1 & 0 \\
\hat{s}_{2 k} & 1
\end{array}\right) .
$$

We define the monodromy data of the system (3.9) by

$$
\left\{\hat{S}_{k}\right\}_{k=1, \ldots, 6}
$$

The behavior (3.13) also implies

$$
\Phi_{7}\left(e^{2 \pi i} z\right)=\Phi_{1}(z) e^{\pi i(2 \mu-1) \sigma_{3}} .
$$

From (3.14)-(3.17), we find the cyclic condition

$$
\hat{S}_{1} \hat{S}_{2} \cdots \hat{S}_{6}=e^{\pi i(2 \mu-1) \sigma_{3}} .
$$

The equation gives us three independent equations of the Stokes multipliers $\hat{s}_{k}, k=1, \cdots, 6$ as given in (3.8); see also [12, Proposition 5.3]. 


\subsection{Reduction of the linear system for PII to THE}

In this subsection, we derive THE from the linear system (1.11) for PII at each pole of the solutions of PII.

Substituting $A(z, x)$ into (1.11) gives

$$
\frac{\mathrm{d}^{2} \Phi_{1}}{\mathrm{~d} z^{2}}-\frac{1}{z-y} \frac{\mathrm{d} \Phi_{1}}{\mathrm{~d} z}-Q(z, x) \Phi_{1}=0,
$$

where

$$
\begin{aligned}
Q(z, x) & =z^{4}+x z^{2}+(1+2 \mu) z+\frac{x^{2}}{4}+v^{2}+\left(2 y^{2}+x\right) v+(1-2 \mu) y-\frac{z^{2}+\frac{x}{2}+v}{z-y} \\
& =z^{4}+x z^{2}+(1+2 \mu) z+\frac{x^{2}}{4}+2 \frac{\mathrm{d}}{\mathrm{d} x} \log \tau(x)-\frac{z^{2}+\frac{x}{2}+v}{z-y} .
\end{aligned}
$$

To eliminate the singularity $z=y$ in (3.19), we consider the poles of $y$. Let $x=a$ be a pole of $y$, then $y$ possesses the following Laurent expansion (see [16, (17.1)]):

$$
y(x)=\frac{\varepsilon}{x-a}-\frac{\varepsilon a}{6}(x-a)-\frac{\mu+\varepsilon}{4}(x-a)^{2}+b(x-a)^{3}+O\left((x-a)^{4}\right),
$$

where $\varepsilon=\varepsilon_{ \pm}= \pm 1$ and $b \in \mathbb{C}$ is arbitrary. From the first equation of the compatibility condition in (3.10) and (3.20), we obtain

$$
v(x)= \begin{cases}-\frac{2}{(x-a)^{2}}+O(1), & \varepsilon=\varepsilon_{+}, \\ O(x-a), & \varepsilon=\varepsilon_{-} .\end{cases}
$$

Hence, taking $x \rightarrow a$ in (3.19) and then making the transformation

$$
\Phi_{1}=w \exp \left(\frac{1}{3} z^{3}+\frac{a}{2} z\right)
$$

we obtain the THEs

$$
\left\{\begin{array}{l}
\frac{\mathrm{d}^{2} w}{\mathrm{~d} z^{2}}+\left(2 z^{2}+a\right) \frac{\mathrm{d} w}{\mathrm{~d} z}+(p z-q) w=0 \\
p=3-2 \mu \\
q=\lim _{x \rightarrow a} 2\left(\frac{\mathrm{d}}{\mathrm{d} x} \log \tau(x)-\frac{1}{x-a}\right)=-\frac{a^{2}}{18}-10 b
\end{array}\right.
$$

for $\varepsilon=\varepsilon_{+}=1$,

$$
\left\{\begin{array}{l}
\frac{\mathrm{d}^{2} w}{\mathrm{~d} z^{2}}+\left(2 z^{2}+a\right) \frac{\mathrm{d} w}{\mathrm{~d} z}+(p z-q) w=0 \\
p=1-2 \mu \\
q=\lim _{x \rightarrow a} 2 \frac{\mathrm{d}}{\mathrm{d} x} \log \tau(x)=-\frac{a^{2}}{18}+10 b
\end{array}\right.
$$

for $\varepsilon=\varepsilon_{-}=-1$. 


\subsection{Isomonodromy set of accessory parameters of THE}

Thus, we see that THE can be obtained as a limit of the first row of the isomonodromy family of $\Phi(z, x)$, when $x \rightarrow a$, with $a$ being the pole of the Painlevé II transcendent corresponding to the same monodromy data of THE. While, the accessory parameters $(a, q)$ are expressed in terms of the parameters in the Laurent expansion of the PII transcendent. Consequently, we have the following description of the isomonodromy set of accessory parameters of THE.

Theorem 2. There is a discrete set of pairs of accessory parameters $\left(a_{n}, q_{n}\right)$ such that the THE (1.5), corresponding to these parameters has the same monodromy data (3.6) and (3.8) as the original equation with the parameters $a$ and $q$. Under the bijection given in the third equation of (3.22), this set coincides with the set of parameters $\left(a_{n}, b_{n}\right)$ in the Laurent expansion (cf. (3.20) near the poles $a_{n}$ of the unique solution of PII equation (3.11) with the parameter $\mu=(3-p) / 2$ and the same monodromy data (3.6) and (3.8) as the THE.

\section{Accessory parameters of RBHE}

\subsection{Monodromy of RBHE}

Consider the RBHE (1.7) with the fixed parameter $2 \alpha$ and the accessory parameters $a$ and $q$. There exist two linear independent solutions $Y_{k}=\left(y_{k 1}, y_{k 2}\right)$ of (1.7) normalizing the asymptotic behavior as $z \rightarrow \infty$

$$
Y_{k}(z) \sim z^{-\left(\alpha+\frac{1}{4}\right)}(1, i) e^{-\left(\frac{2}{3} z^{\frac{3}{2}}+a z^{\frac{1}{2}}\right) \sigma_{3}}, \quad z \in \Omega_{k},
$$

where $\Omega_{k}$ for $k=-1, \cdots, 2$ are the Stokes sectors

$$
\Omega_{k}=\left\{z \in \mathbb{C}: \frac{\pi}{3}(2 k-3)<\arg z<\frac{\pi}{3}(2 k+1)\right\} .
$$

The solutions are related to each other by the Stokes matrices

$$
Y_{k+1}(z)=Y_{k}(z) S_{k}, \quad k=-1,0,1,
$$

where

$$
S_{ \pm 1}=\left(\begin{array}{cc}
1 & 0 \\
s_{ \pm 1} & 1
\end{array}\right), \quad S_{0}=\left(\begin{array}{cc}
1 & s_{0} \\
0 & 1
\end{array}\right)
$$

The behavior (4.1) also gives

$$
Y_{2}\left(e^{2 \pi i} z\right)=e^{-2 \pi i \alpha} Y_{-1}(z) i \sigma_{2} .
$$

Moreover, from (1.7) we see that the characteristic exponents at $z=0$ are $\{0,1-2 \alpha\}$. Hence, as $z \rightarrow 0$, we have the asymptotic behavior

$$
Y_{2}(z) \sim z^{-\alpha}(1, z) z^{\alpha \sigma_{3}} E
$$

for $2 \alpha \notin \mathbb{Z}$, with $E$ being some invertible constant matrix. Using (4.3)-(4.6), we obtain the same cyclic condition as that for the Lax pair of the PXXXIV equation

$$
S_{-1} S_{0} S_{1}=i \sigma_{2} E^{-1} e^{-2 \pi i \alpha \sigma_{3}} E .
$$

This condition implies the following algebraic equation

$$
s_{-1} s_{0} s_{1}+s_{-1}+s_{1}-s_{0}=-2 \cos (2 \pi \alpha) \text {. }
$$

Thus, the monodromy data for RBHE (1.7) are described by the 2-dimensional complex surface

$$
\left\{\left(s_{-1}, s_{0}, s_{1}\right) \in \mathbb{C}^{3}: s_{-1} s_{0} s_{1}+s_{-1}+s_{1}-s_{0}=-2 \cos (2 \pi \alpha)\right\} .
$$




\subsection{Isomonodromy deformation and PXXXIV equation}

To study the isomonodromy deformation of the RBHE (1.7), we regard $z^{\alpha} Y_{k}(z)$ as the first row of a $2 \times 2$ matrix system $\Phi_{k}(z, x)$ when $x \rightarrow a$. Then, we look for the system $\Phi_{k}(z, x)$ such that $\Phi_{k}(z, x)$ satisfy the same jump relations (4.3)-(4.5) and the following asymptotic behaviors

$$
\Phi_{k}(z, x)=z^{-\frac{1}{4} \sigma_{3}} \frac{I+i \sigma_{1}}{\sqrt{2}}\left(I+O\left(z^{-\frac{1}{2}}\right)\right) e^{-\left(\frac{2}{3} z^{\frac{3}{2}}+x z^{\frac{1}{2}}\right) \sigma_{3}}, \quad z \in \Omega_{k},
$$

as $z \rightarrow \infty$, where $\Omega_{k}, k=-1, \cdots, 2$, are the Stokes sectors defined in (4.2), and

$$
\Phi_{2}(z, x)=\Phi^{(0)}(x)(I+O(z)) z^{\alpha \sigma_{3}} E, \quad z \rightarrow 0,
$$

where the parameter $2 \alpha \notin \mathbb{Z}$ and $\Phi^{(0)}(x)$ is a matrix independent of $z$. The behavior of $\Phi_{k}(z, x)$ as $z \rightarrow 0$ are then determined by (4.10) and the jump relations.

It can be shown that $\Phi_{k}(z, x)$ satisfies the following Lax pair with rational coefficients (see [12, Chapter 5], [18, Lemma 3.2] and [34])

$$
\left\{\begin{array}{l}
\frac{\partial \Phi(z, x)}{\partial z}=A(z, x) \Phi(z, x) \\
\frac{\partial \Phi(z, x)}{\partial x}=B(z, x) \Phi(z, x)
\end{array}\right.
$$

where

$$
A(z, x)=\left(\begin{array}{cc}
\frac{y^{\prime}}{2 z} & i-i \frac{y}{z} \\
-i z-i(y+x)-i \frac{\left(y^{\prime}\right)^{2}-(2 \alpha)^{2}}{4 y z} & -\frac{y^{\prime}}{2 z}
\end{array}\right), \quad B(z, x)=\left(\begin{array}{cc}
0 & i \\
-i z-2 i\left(y+\frac{x}{2}\right) & 0
\end{array}\right) .
$$

The Lax pair is related by a gauge transformation to the Lax pair for PII found by Flaschka and Newell; see for instance [12, Chapter 5] and [18, Equation (3.19)].

The compatibility condition of the above Lax pair is described by the PXXXIV equation

$$
\frac{\mathrm{d}^{2} y}{\mathrm{~d} x^{2}}=\frac{1}{2 y}\left(\frac{\mathrm{d} y}{\mathrm{~d} x}\right)^{2}+4 y^{2}+2 x y-\frac{(2 \alpha)^{2}}{2 y} .
$$

Let

$$
v(x)=\frac{y^{\prime}(x)-2 \alpha}{2 y(x)},
$$

then $-2^{-1 / 3} v\left(-2^{-1 / 3} x\right)$ satisfies the PII equation (3.11) with $\mu=-\left(2 \alpha+\frac{1}{2}\right)$. The associated $\tau$-function can be defined as

$$
\frac{\mathrm{d}}{\mathrm{d} x} \log \tau(x)=-y(x)^{2}+\left(v(x)^{2}-x\right) y(x)+2 \alpha v(x),
$$

which is related simply to the standard Hamiltonian for the PII equation; cf. [25, Equation (32.6.9)].

Thus, we see that the solutions $\Phi_{k}(z, x)$ to the Lax pair (4.12) subject to the boundary conditions (4.10)-(4.11) share the same monodromy data as RBHE (independent of $x$ ) given in (4.9). While the isomonodromy deformation of $\Phi_{k}(z, x)$ with respect to $x$ are described by the PXXXIV equation.

In the next subsection, we show that the RBHE can actually be obtained as a limit of the linear system (1.11), associated with the Lax pair for the Painlevé XXXIV equation, when the Painlevé XXXIV transcendent $y(x)$ tends to zero or infinity. 


\subsection{Reduction of the linear system for PXXXIV to RBHE}

Substituting the elements of $A(z, x)$ into (1.11) gives

$$
\frac{\mathrm{d}^{2} \Phi_{1}}{\mathrm{~d} z^{2}}-\left(\frac{1}{z-y}-\frac{1}{z}\right) \frac{\mathrm{d} \Phi_{1}}{\mathrm{~d} z}-P(z, x) \Phi_{1}=0,
$$

where

$$
\begin{aligned}
P(z, x) & =z+x+\frac{\left(\left(y^{\prime}\right)^{2}-4 \alpha^{2}\right) /(4 y)-y^{2}-x y}{z}+\frac{\alpha^{2}}{z^{2}}-\frac{y^{\prime}}{2 z(z-y)} \\
& =z+x+\frac{1}{z}\left(\frac{\mathrm{d}}{\mathrm{d} x} \log \tau(x)\right)+\frac{\alpha^{2}}{z^{2}}-\frac{y^{\prime}}{2 z(z-y)} .
\end{aligned}
$$

To eliminate the singularity $z=y$, we consider the poles and zeros of $y$. It is readily seen that $y$ solving (4.13) possesses the following Laurent expansion at a pole

$$
y(x)=\frac{1}{(x-a)^{2}}-\frac{a}{3}-\frac{1}{2}(x-a)+b(x-a)^{2}+O\left((x-a)^{3}\right),
$$

and the Taylor expansion at a zero

$$
y(x)=\varepsilon(x-a)+b(x-a)^{2}+\frac{2 a \varepsilon}{3}(x-a)^{3}+O\left((x-a)^{4}\right),
$$

where $\varepsilon=\varepsilon_{ \pm}= \pm 2 \alpha$ and $b$ is arbitrary.

Taking $x \rightarrow a$ in (4.15) and making the transformation

$$
\Phi_{1}=z^{\alpha} w,
$$

we see that $w(z)$ satisfies the following RBHEs

$$
\left\{\begin{array}{l}
\frac{\mathrm{d}^{2} w}{\mathrm{~d} z^{2}}+\frac{2 \alpha+1}{z} \frac{\mathrm{d} w}{\mathrm{~d} z}-\left(z+a+\frac{q}{z}\right) w=0 \\
q=\lim _{x \rightarrow a}\left(\frac{\mathrm{d}}{\mathrm{d} x} \log \tau(x)-\frac{1}{x-a}\right)=\frac{a^{2}}{3}-5 b
\end{array}\right.
$$

when $a$ is a pole of $y(x)$, and

$$
\left\{\begin{array}{l}
\frac{\mathrm{d}^{2} w}{\mathrm{~d} z^{2}}+\frac{2 \alpha}{z} \frac{\mathrm{d} w}{\mathrm{~d} z}-\left(z+a+\frac{q}{z}\right) w=0, \\
q=\lim _{x \rightarrow a} \frac{\mathrm{d}}{\mathrm{d} x} \log \tau(x)=b
\end{array}\right.
$$

when $a$ is a zero of $y(x)$ with coefficient $\varepsilon=\varepsilon_{+}=2 \alpha$ in (4.17). When $a$ is a zero with coefficient $\varepsilon=\varepsilon_{-}=-2 \alpha$, taking $x \rightarrow a$ and the transformation $\Phi_{1}=z^{\alpha+1} w$ in (4.15) we arrive at

$$
\left\{\begin{array}{l}
\frac{\mathrm{d}^{2} w}{\mathrm{~d} z^{2}}+\frac{2(\alpha+1)}{z} \frac{\mathrm{d} w}{\mathrm{~d} z}-\left(z+a+\frac{q}{z}\right) w=0 \\
q=\lim _{x \rightarrow a} \frac{\mathrm{d}}{\mathrm{d} x} \log \tau(x)=b .
\end{array}\right.
$$




\subsection{Isomonodromy set of accessory parameters of RBHE}

Thus, we see that RBHE can be obtained as a limit of the first row of the isomonodromy family of $\Phi(z, x)$ when $x \rightarrow a, a$ being a zero or pole of the Painlevé XXXIV transcendents corresponding to the same monodromy data as RBHE. While the accessory parameters $(a, q)$ are expressed in terms of the parameters in the Taylor or Laurent expansion of the PXXXIV transcendents. As a consequence, we have the following description of the isomonodromy accessory parameters set of RBHE.

Theorem 3. There is a discrete set of pairs of accessory parameters $\left(a_{n}, q_{n}\right)$ such that the RBHE (1.7) corresponding to these parameters has the same monodromy data (4.9) as the original equation with the parameters $a$ and $q$. This set coincides with the set of parameters $\left(a_{n}, b_{n}\right)$ in the Taylor expansion (4.17) near the zeros $a_{n}$ of the unique solution of the PXXXIV equation (4.13) with the same monodromy data (4.9) as the RBHE.

\section{Accessory parameters of DHE}

\subsection{Monodromy of DHE}

Consider the DHE (1.3) with the accessory parameters $a$ and $q$ and the fixed parameters $\gamma$ and $p$. Let $\Omega_{k}^{(\infty)}, k=1,2$, be the Stokes sectors

$$
\Omega_{k}^{(\infty)}=\left\{z \in \mathbb{C}: \frac{\pi}{2}(-2 k+1)<\arg z<\frac{\pi}{2}(-2 k+5)\right\} .
$$

There exist unique linear independent solutions of (1.3), namely $Y_{k}^{(\infty)}=\left(y_{k 1}^{(\infty)}, y_{k 2}^{(\infty)}\right)$, which satisfy the normalized asymptotic behavior as $z \rightarrow \infty$

$$
Y_{k}^{(\infty)}(z) \sim e^{-\frac{z}{4}} z^{-\frac{1}{2} \theta_{0}}(1,1 / z) e^{\frac{z}{4} \sigma_{3}} z^{-\frac{1}{2} \theta_{\infty} \sigma_{3}}, \quad z \in \Omega_{k}^{(\infty)},
$$

for $k=1,2$. Here the parameters $\theta_{0}=\gamma-1$ and $\theta_{\infty}=4 p-\gamma+1$. The solutions are related to each other through the Stokes matrices

$$
\begin{gathered}
Y_{2}^{(\infty)}(z)=Y_{1}^{(\infty)}(z) S_{1}^{(\infty)}, \quad S_{1}^{(\infty)}=\left(\begin{array}{cc}
1 & 0 \\
s_{1}^{(\infty)} & 1
\end{array}\right) \\
Y_{1}^{(\infty)}\left(z e^{2 \pi i}\right) e^{\pi i \theta_{0}} e^{\pi i \theta_{\infty} \sigma_{3}}=Y_{2}^{(\infty)}(z) S_{2}^{(\infty)}, \quad S_{2}^{(\infty)}=\left(\begin{array}{cc}
1 & s_{2}^{(\infty)} \\
0 & 1
\end{array}\right) .
\end{gathered}
$$

Parallelly, there exist unique linear independent solutions of (1.3) determined by the asymptotic behavior as $z \rightarrow 0$

$$
Y_{k}^{(0)}(z) \sim e^{-\frac{a^{2}}{4 z}} z^{-\frac{1}{2} \theta_{0}}(1, z) e^{\frac{a^{2}}{4 z} \sigma_{3}} z^{\frac{1}{2} \theta_{0} \sigma_{3}}, \quad z \in \Omega_{k}^{(0)},
$$

where $\Omega_{k}^{(0)}$ for $k=1,2$ are the Stokes sectors

$$
\Omega_{k}^{(0)}=\left\{z \in \mathbb{C}: \frac{\pi}{2}(-2 k+1)<\arg \left(a^{2} / z\right)<\frac{\pi}{2}(-2 k+5)\right\} .
$$

The solutions are related to each other by the Stokes matrices

$$
Y_{2}^{(0)}(z)=Y_{1}^{(0)}(z) S_{1}^{(0)}, \quad S_{1}^{(0)}=\left(\begin{array}{cc}
1 & 0 \\
s_{1}^{(0)} & 1
\end{array}\right)
$$




$$
Y_{1}^{(0)}\left(z e^{-2 \pi i}\right) e^{-\pi i \theta_{0}} e^{\pi i \theta_{0} \sigma_{3}}=Y_{2}^{(0)}(z) S_{2}^{(0)}, \quad S_{2}^{(0)}=\left(\begin{array}{cc}
1 & s_{2}^{(0)} \\
0 & 1
\end{array}\right) .
$$

Moreover, the solutions $Y_{1}^{(\infty)}(z)$ and $Y_{2}^{(0)}(z)$ are connected by

$$
Y_{1}^{(\infty)}(z)=Y_{2}^{(0)}(z) E
$$

where $E$ is some invertible constant matrix. We define the monodromy data of (1.3) by the set

$$
\left\{e^{\pi i \theta_{\infty}}, e^{\pi i \theta_{0}} ; S_{1}^{(\infty)}, S_{2}^{(\infty)} ; S_{1}^{(0)}, S_{2}^{(0)} ; E\right\}
$$

Combining (5.3), (5.4), and (5.7)-(15.9), we have the cyclic condition

$$
S_{1}^{(\infty)} S_{2}^{(\infty)} e^{-\pi i \theta_{\infty} \sigma_{3}}=E^{-1}\left(S_{1}^{(0)}\right)^{-1} e^{\pi i \theta_{0} \sigma_{3}}\left(S_{2}^{(0)}\right)^{-1} E .
$$

The condition implies that the Stokes multipliers satisfy the equation

$$
e^{\pi i \theta_{\infty}} s_{1}^{(\infty)} s_{2}^{(\infty)}+2 \cos \left(\pi \theta_{\infty}\right)=e^{\pi i \theta_{0}} s_{1}^{(0)} s_{2}^{(0)}+2 \cos \left(\pi \theta_{0}\right) .
$$

\subsection{Isomonodromy deformation and PIII equation}

To study the isomonodromy deformation of the RTHE equation, it is convenient to consider a $2 \times 2$ matrix system, which has two irregular singular points, both of rank 1 , at infinity and at the origin. The isomonodromy deformation of a matrix system with such a singular pattern has been considered in [12, 20].

Recall the following Lax pair for PIII (see [20, (C.18)-(C.20)]):

$$
\left\{\begin{array}{l}
\frac{\partial \Phi(z, x)}{\partial z}=A(z, x) \Phi(z, x) \\
\frac{\partial \Phi(z, x)}{\partial x}=B(z, x) \Phi(z, x)
\end{array}\right.
$$

where

$$
\begin{aligned}
& A(z, x)=\left(\begin{array}{cc}
\frac{x}{4}-\frac{\theta_{\infty}}{2 z}+\frac{4 v-x}{4 z^{2}} & \frac{r}{z}-\frac{u v}{z^{2}} \\
\frac{s}{z}+\frac{2 v-x}{2 u z^{2}} & -\frac{x}{4}+\frac{\theta_{\infty}}{2 z}-\frac{4 v-x}{4 z^{2}}
\end{array}\right), \\
& B(z, x)=\left(\begin{array}{ll}
\frac{z}{4}-\frac{4 v-x}{4 x z} & \frac{r}{x}+\frac{u v}{x z} \\
\frac{s}{x}-\frac{2 v-x}{2 x u z} & -\frac{z}{4}+\frac{4 v-x}{4 x z}
\end{array}\right) .
\end{aligned}
$$

If we set $y=-r / u v$ and

$$
\frac{\theta_{0}}{2}=\frac{v}{x}\left[\frac{\theta_{\infty}}{2}\left(4-\frac{x}{v}\right)+\frac{x-2 v}{u v} r+2 u s\right]
$$

then the compatibility condition of the above Lax pair reads

$$
\left\{\begin{array}{l}
x \frac{\mathrm{d} y}{\mathrm{~d} x}=(4 v-x) y^{2}+\left(2 \theta_{\infty}-1\right) y+x \\
x \frac{\mathrm{d} v}{\mathrm{~d} x}=-4 y v^{2}+\left(2 x y-2 \theta_{\infty}+1\right) v+\frac{1}{2}\left(\theta_{0}+\theta_{\infty}\right) x \\
x \frac{\mathrm{d} u}{\mathrm{~d} x}=u\left(-\frac{x}{2 v}\left(\theta_{0}+\theta_{\infty}\right)-x y+\theta_{\infty}\right)
\end{array}\right.
$$


which implies the PIII equation

$$
\frac{\mathrm{d}^{2} y}{\mathrm{~d} x^{2}}=\frac{1}{y}\left(\frac{\mathrm{d} y}{\mathrm{~d} x}\right)^{2}-\frac{1}{x} \frac{\mathrm{d} y}{\mathrm{~d} x}+\frac{2 \theta_{0} y^{2}+2-2 \theta_{\infty}}{x}+y^{3}-\frac{1}{y} .
$$

The PIII $\tau$-function is defined by (see [20, (C.27)])

$$
x \frac{\mathrm{d}}{\mathrm{d} x} \log \tau(x)=2 y^{2} v^{2}+\left(-x y^{2}+2 \theta_{\infty} y+x\right) v-\frac{\left(\theta_{0}+\theta_{\infty}\right) x y}{2}-\frac{x^{2}}{4}-\frac{\theta_{0}^{2}-\theta_{\infty}^{2}}{4} .
$$

Using (5.14) and (5.15) to eliminate $v(x)$, we have

$$
x \frac{\mathrm{d}}{\mathrm{d} x} \log \tau(x)=\frac{x^{2} y^{\prime 2}}{8 y^{2}}+\frac{x y^{\prime}}{4 y}-\frac{x^{2}}{8 y^{2}}-\frac{x^{2} y^{2}}{8}-\frac{\theta_{\infty} x}{2 y}-\frac{\theta_{0} x y}{2}+\frac{1}{8}-\frac{\theta_{0}^{2}+\theta_{\infty}^{2}}{4} .
$$

There exist unique solutions $\Phi_{k}^{(\infty)}(z, x)$ of the equation (5.13), which satisfy the normalized asymptotic behavior as $z \rightarrow \infty$

$$
\Phi_{k}^{(\infty)}(z, x)=(I+O(1 / z)) e^{\frac{x z}{4} \sigma_{3}} z^{-\frac{1}{2} \theta_{\infty} \sigma_{3}}, \quad x z \in \Omega_{k}^{(\infty)},
$$

where $\Omega_{k}^{(\infty)}$ for $k=1,2$ are the Stokes sectors defined in (5.1). The solutions are related to each other by the Stokes matrices

$$
\begin{gathered}
\Phi_{2}^{(\infty)}(z)=\Phi_{1}^{(\infty)}(z) \hat{S}_{1}^{(\infty)}, \quad \hat{S}_{1}^{(\infty)}=\left(\begin{array}{cc}
1 & 0 \\
\hat{s}_{1}^{(\infty)} & 1
\end{array}\right), \\
\Phi_{1}^{(\infty)}\left(z e^{2 \pi i}\right) e^{\pi i \theta_{\infty} \sigma_{3}}=\Phi_{2}^{(\infty)}(z) \hat{S}_{2}^{(\infty)}, \quad \hat{S}_{2}^{(\infty)}=\left(\begin{array}{cc}
1 & \hat{s}_{2}^{(\infty)} \\
0 & 1
\end{array}\right) .
\end{gathered}
$$

Similarly, there exist unique solutions $\Phi_{k}^{(0)}(z, x)$ of the equation (5.13) such that

$$
\Phi_{k}^{(0)}(z, x)=G_{k}(x)(I+O(z)) e^{\frac{x}{4 z} \sigma_{3}} z^{\frac{1}{2} \theta_{0} \sigma_{3}}, \quad z \in \Omega_{k}^{(0)}, \quad z \rightarrow 0,
$$

where $G_{k}(x)$ are matrices independent of $z$, and the sectors $\Omega_{k}^{(0)}, k=1,2$, are defined in (5.6) with $\arg \left(a^{2} / z\right)$ replaced by $\arg (x / z)$. These solutions are related to each other by the Stokes matrices

$$
\begin{gathered}
\Phi_{2}^{(0)}(z)=\Phi_{1}^{(0)}(z) \hat{S}_{1}^{(0)}, \quad \hat{S}_{1}^{(0)}=\left(\begin{array}{cc}
1 & 0 \\
\hat{s}_{1}^{(0)} & 1
\end{array}\right) \\
\Phi_{1}^{(0)}\left(z e^{-2 \pi i}\right) e^{\pi i \theta_{0} \sigma_{3}}=\Phi_{2}^{(0)}(z) \hat{S}_{2}^{(0)}, \quad \hat{S}_{2}^{(0)}=\left(\begin{array}{cc}
1 & \hat{s}_{2}^{(0)} \\
0 & 1
\end{array}\right) .
\end{gathered}
$$

Moreover, the solutions $\Phi_{1}^{(\infty)}(z)$ and $\Phi_{2}^{(0)}(z)$ are connected through

$$
\Phi_{1}^{(\infty)}(z)=\Phi_{2}^{(0)}(z) \hat{E}
$$

where $\hat{E}$ is some invertible constant matrix. The monodromy data of the system (5.13) is defined as the set

$$
\left\{e^{\pi i \theta_{\infty}}, e^{\pi i \theta_{0}} ; \hat{S}_{1}^{(\infty)}, \hat{S}_{2}^{(\infty)} ; \hat{S}_{1}^{(0)}, \hat{S}_{2}^{(0)} ; \hat{E}\right\}
$$

Applying (5.18), (5.19), and (5.21)-(5.23), we find the cyclic condition for the monodromy data

$$
\hat{S}_{1}^{(\infty)} \hat{S}_{2}^{(\infty)} e^{-\pi i \theta_{\infty} \sigma_{3}}=\hat{E}^{-1}\left(\hat{S}_{1}^{(0)}\right)^{-1} e^{\pi i \theta_{0} \sigma_{3}}\left(\hat{S}_{2}^{(0)}\right)^{-1} \hat{E} .
$$

Thus, the Stokes multipliers satisfy the equation

$$
e^{\pi i \theta_{\infty}} \hat{s}_{1}^{(\infty)} \hat{s}_{2}^{(\infty)}+2 \cos \left(\pi \theta_{\infty}\right)=e^{\pi i \theta_{0}} \hat{s}_{1}^{(0)} \hat{s}_{2}^{(0)}+2 \cos \left(\pi \theta_{0}\right) .
$$




\subsection{Reduction of the linear system for PIII to DHE}

In this section, we will show that the DHE (1.3) can be obtained as a limit of the linear system (1.11) associated with the Lax pair for the Painlevé III equation as $x \rightarrow a$, where $a$ is one of the poles or zeros of the solution to the Painlevé III equation.

Substituting the elements of $A(z, x)$ into (1.11) gives

$$
\frac{\mathrm{d}^{2} \Phi_{1}}{\mathrm{~d} z^{2}}-\left(\frac{1}{z+1 / y}-\frac{2}{z}\right) \frac{\mathrm{d} \Phi_{1}}{\mathrm{~d} z}-Q(z, x) \Phi_{1}=0,
$$

where

$$
\begin{aligned}
Q(z, x)= & \frac{x^{2}}{16}+\frac{\left(2-\theta_{\infty}\right) x}{4 z}-\frac{\theta_{0} x}{4 z^{3}}+\frac{x^{2}}{16 z^{4}}-\frac{1}{z+1 / y}\left(\frac{x}{4}-\frac{\theta_{\infty}}{2 z}+\frac{v-\frac{x}{4}}{z^{2}}\right) \\
& +\frac{1}{z^{2}}\left[y^{2} v^{2}+\frac{1}{2}\left(-x y^{2}+2 \theta_{\infty} y+x\right) v-\frac{1}{4}\left(\theta_{0}+\theta_{\infty}\right) x y-\frac{x^{2}}{8}+\frac{\theta_{\infty}^{2}}{4}-\frac{\theta_{\infty}}{2}\right] \\
= & \frac{x^{2}}{16}+\frac{\left(2-\theta_{\infty}\right) x}{4 z}-\frac{\theta_{0} x}{4 z^{3}}+\frac{x^{2}}{16 z^{4}}-\frac{1}{z+1 / y}\left(\frac{x}{4}-\frac{\theta_{\infty}}{2 z}+\frac{v-\frac{x}{4}}{z^{2}}\right) \\
& +\frac{1}{z^{2}}\left[\frac{1}{2} x \frac{\mathrm{d}}{\mathrm{d} x} \log \tau(x)+\frac{\theta_{0}^{2}+\theta_{\infty}^{2}}{8}-\frac{\theta_{\infty}}{2}\right] .
\end{aligned}
$$

To obtain DHE, we eliminate the extra singularity $z=-1 / y$ in the above equation by considering the poles and zeros of $y$. It is known that $y$ admits the following Laurent expansion near a pole

$$
y(x)=\frac{\varepsilon}{x-a}-\frac{2 \theta_{0}+\varepsilon}{2 a}+b(x-a)+O\left((x-a)^{2}\right),
$$

and the Taylor expansion near a zero

$$
y(x)=\sigma(x-a)+\frac{\sigma-2+2 \theta_{\infty}}{2 a}(x-a)^{2}+b(x-a)^{3}+O\left((x-a)^{4}\right),
$$

where $\varepsilon=\varepsilon_{ \pm}= \pm 1, \sigma=\sigma_{ \pm}= \pm 1, a \neq 0$ and $b$ is arbitrary.

In view of the first equation of compatibility condition (5.14), (5.28) and (5.29), we obtain that

$$
v(x)= \begin{cases}O(x-a), & \varepsilon=\varepsilon_{+}, \\ \frac{a}{2}+O(x-a), & \varepsilon=\varepsilon_{-}, \\ O(1), & \sigma=\sigma_{+}, \\ -\frac{a}{2}(x-a)^{-2}+O(1), & \sigma=\sigma_{-} .\end{cases}
$$

Thus, taking $x \rightarrow a$ in (5.27) and setting

$$
\Phi_{1}=z^{\frac{\theta_{0}}{2}} e^{\frac{a}{4}\left(z+\frac{1}{z}\right)} w, \quad s=a z,
$$

we obtain the DHEs

$$
\left\{\begin{array}{l}
\frac{\mathrm{d}^{2} w}{\mathrm{~d} s^{2}}+\left(\frac{1}{2}+\frac{1+\theta_{0}}{s}-\frac{a^{2}}{2 s^{2}}\right) \frac{\mathrm{d} w}{\mathrm{~d} s}+\frac{p s-q}{s^{2}} w=0 \\
p=\frac{1}{4}\left(\theta_{\infty}+\theta_{0}\right) \\
q=\lim _{x \rightarrow a} \frac{1}{2} x \frac{\mathrm{d}}{\mathrm{d} x} \log \tau(x)+\frac{a^{2}}{8}-\frac{\theta_{0}^{2}-\theta_{\infty}^{2}}{8} \\
=-\frac{3}{8} a^{2} b+\frac{a^{2}}{8}+\frac{1}{32}\left(2 \theta_{0}+1\right)\left(2 \theta_{0}+5\right)
\end{array}\right.
$$


for $\varepsilon=\varepsilon_{+}$,

$$
\left\{\begin{array}{l}
\frac{\mathrm{d}^{2} w}{\mathrm{~d} s^{2}}+\left(\frac{1}{2}+\frac{2+\theta_{0}}{s}-\frac{a^{2}}{2 s^{2}}\right) \frac{\mathrm{d} w}{\mathrm{~d} s}+\frac{p s-q}{s^{2}} w=0 \\
p=\frac{1}{4}\left(\theta_{\infty}+\theta_{0}\right), \\
q=\lim _{x \rightarrow a} \frac{1}{2} x \frac{\mathrm{d}}{\mathrm{d} x} \log \tau(x)+\frac{a^{2}}{8}-\frac{\theta_{0}^{2}-\theta_{\infty}^{2}}{8}-\frac{\theta_{0}+\theta_{\infty}}{2}
\end{array}\right.
$$

for $\sigma=\sigma_{+}$, and

$$
\left\{\begin{array}{l}
\frac{\mathrm{d}^{2} w}{\mathrm{~d} s^{2}}+\left(\frac{1}{2}+\frac{2+\theta_{0}}{s}-\frac{a^{2}}{2 s^{2}}\right) \frac{\mathrm{d} w}{\mathrm{~d} s}+\frac{p s-q}{s^{2}} w=0, \\
p=\frac{1}{4}\left(\theta_{\infty}+\theta_{0}+2\right), \\
q=\lim _{x \rightarrow a} \frac{1}{2}\left(x \frac{\mathrm{d}}{\mathrm{d} x} \log \tau(x)-\frac{a}{x-a}\right)+\frac{a^{2}}{8}-\frac{\theta_{0}^{2}-\theta_{\infty}^{2}}{8}-\frac{\theta_{0}}{2}-\frac{3}{4}
\end{array}\right.
$$

for $\sigma=\sigma_{-}$. For $\varepsilon=\varepsilon_{-}$in (5.27), taking $x \rightarrow a$ and setting

$$
\Phi_{1}=z^{-\frac{\theta_{0}}{2}} e^{\frac{a}{4}\left(z-\frac{1}{z}\right)} w, \quad s=a z,
$$

we obtain the DHE

$$
\left\{\begin{array}{l}
\frac{\mathrm{d}^{2} w}{\mathrm{~d} s^{2}}+\left(\frac{1}{2}+\frac{1-\theta_{0}}{s}+\frac{a^{2}}{2 s^{2}}\right) \frac{\mathrm{d} w}{\mathrm{~d} s}+\frac{p s-q}{s^{2}} w=0 \\
p=\frac{1}{4}\left(\theta_{\infty}-\theta_{0}\right) \\
q=\lim _{x \rightarrow a} \frac{1}{2} x \frac{\mathrm{d}}{\mathrm{d} x} \log \tau(x)-\frac{a^{2}}{8}-\frac{\theta_{0}^{2}-\theta_{\infty}^{2}}{8} .
\end{array}\right.
$$

\subsection{Isomonodromy set of accessory parameters of DHE}

Consider the DHE (1.3) with the accessory parameters $a$ and $q$ and the fixed parameters $\gamma$ and $p$. We have shown that the DHE can be obtained as a limit of the first row of the isomonodromy family of $\Phi(z, x)$, when $x$ tends to one of the poles or zeros of the Painlevé III transcendents corresponding to the same monodromy data of the DHE. While the accessory parameters $(a, q)$ are expressed in terms of the parameters in the Laurent or Taylor expansion of the PIII transcendents with the parameters $\theta_{0}=\gamma-1$ and $\theta_{\infty}=4 p-\gamma+1$. As a result, we have the following description of the isomonodromy set of accessory parameters of DHE.

Theorem 4. There is a discrete set of pairs of accessory parameters $\left(a_{n}, q_{n}\right)$ such that the DHE (1.3) corresponding to these parameters has the same monodromy data as the original equation with the parameters a and $q$. Under the bijection given in the last equation of (5.31), this set coincides with the set of parameters $\left(a_{n}, b_{n}\right)$ in the Laurent expansion near the poles $a_{n}$ of the unique solution of PIII with the same monodromy data (5.10) as the DHE.

\section{Accessory parameters of BHE}

\subsection{Monodromy of BHE}

Consider the BHE (1.4) with the accessory parameters $a$ and $q$, the other fixed parameters $\gamma$ and $p$. There exist unique solutions of (1.4) which satisfy the normalized asymptotic behavior 
as $z \rightarrow \infty$

$$
Y_{k}(z) \sim e^{-\frac{z^{2}}{2}-a z} z^{-\left(\theta_{0}+1\right)}(1,1) e^{\frac{z^{2}+2 a z}{2} \sigma_{3}} z^{-\theta_{\infty} \sigma_{3}}, \quad z \in \Omega_{k},
$$

where the parameters $\theta_{0}=(\gamma-1) / 2, \theta_{\infty}=(p-\gamma-1) / 2$ and the Stokes sectors $\Omega_{k}, k=1,2,3,4$ are given by

$$
\Omega_{k}=\left\{z \in \mathbb{C}: \frac{\pi}{4}(2 k-5)<\arg z<\frac{\pi}{4}(2 k-1)\right\} .
$$

The solutions are related to each other by the Stokes matrices

$$
\begin{gathered}
Y_{k+1}(z)=Y_{k}(z) S_{k}, \quad k=1,2,3, \\
Y_{1}(z)=Y_{4}\left(z e^{2 \pi i}\right) S_{4} e^{2 \pi i \theta_{0}} e^{2 \pi i \theta_{\infty} \sigma_{3}},
\end{gathered}
$$

where

$$
S_{2 k-1}=\left(\begin{array}{cc}
1 & 0 \\
s_{2 k-1} & 1
\end{array}\right), \quad S_{2 k}=\left(\begin{array}{cc}
1 & s_{2 k} \\
0 & 1
\end{array}\right), \quad k=1,2 .
$$

The solution $Y_{1}(z)$ has the asymptotic behavior as $z \rightarrow 0$

$$
Y_{1}(z) \sim z^{-\theta_{0}}(1,1) z^{\theta_{0} \sigma_{3}} E,
$$

with some invertible constant matrix $E$. The monodromy data of BHE (1.4) is defined as the set

$$
\left\{e^{2 \pi i \theta_{\infty}}, e^{2 \pi i \theta_{0}} ; S_{1}, S_{2}, S_{3}, S_{4} ; E\right\}
$$

By (6.3) $-(\underline{6.6})$, they satisfy the cyclic condition

$$
S_{1} S_{2} S_{3} S_{4} e^{2 \pi i \theta_{\infty} \sigma_{3}}=E^{-1} e^{-2 \pi i \theta_{0} \sigma_{3}} E .
$$

Thus, the Stokes multipliers $s_{k}, k=1,2,3,4$, satisfy the algebraic equation

$$
e^{2 \pi i \theta_{\infty}}\left(1+s_{2} s_{3}\right)+e^{-2 \pi i \theta_{\infty}}\left[s_{1} s_{4}+\left(1+s_{1} s_{2}\right)\left(1+s_{3} s_{4}\right)\right]=2 \cos \left(2 \pi \theta_{0}\right) .
$$

It is direct to see that the cyclic condition (6.8) also specifies the connection matrix $E$ to within a left-multiplicative diagonal matrix.

\subsection{Isomonodromy deformation and PIV equation}

To study the isomonodromy deformation of the BHE, it is convenient to consider a $2 \times 2$ matrix system, which has one irregular singular point of rank 2 at infinity and one regular singularity at the origin. In [12, 20], the isomonodromy deformation of such a matrix system has been considered.

Consider the following Lax pair for PIV (see [20, (C.30)-(C.31)]):

$$
\left\{\begin{array}{l}
\frac{\partial \Phi(z, x)}{\partial z}=A(z, x) \Phi(z, x) \\
\frac{\partial \Phi(z, x)}{\partial x}=B(z, x) \Phi(z, x)
\end{array}\right.
$$

where

$$
\begin{array}{ll}
A(z, x) & =\left(\begin{array}{cc}
z+x+\frac{\theta_{0}-v}{z} & u\left(1-\frac{y}{2 z}\right) \\
\frac{2}{u}\left(v-\theta_{0}-\theta_{\infty}+\frac{v^{2}-2 \theta_{0} v}{y z}\right) & -z-x-\frac{\theta_{0}-v}{z}
\end{array}\right), \\
B(z, x) & =\left(\begin{array}{cc}
z & u \\
\frac{2}{u}\left(v-\theta_{0}-\theta_{\infty}\right) & -z
\end{array}\right) .
\end{array}
$$


The compatibility condition of the Lax pair gives

$$
\left\{\begin{array}{l}
\frac{\mathrm{d} u}{\mathrm{~d} x}=-u(y+2 x) \\
\frac{\mathrm{d} y}{\mathrm{~d} x}=-4 v+y^{2}+2 x y+4 \theta_{0} \\
\frac{\mathrm{d} v}{\mathrm{~d} x}=-\left(v-\theta_{0}-\theta_{\infty}\right) y-\frac{2 v\left(v-2 \theta_{0}\right)}{y}
\end{array}\right.
$$

which leads to the PIV equation

$$
\frac{\mathrm{d}^{2} y}{\mathrm{~d} x^{2}}=\frac{1}{2 y}\left(\frac{\mathrm{d} y}{\mathrm{~d} x}\right)^{2}+\frac{3}{2} y^{3}+4 x y^{2}+2\left(x^{2}+1-2 \theta_{\infty}\right) y-\frac{8 \theta_{0}^{2}}{y} .
$$

The PIV $\tau$-function is defined by (see [20, (C.35)])

$$
\frac{\mathrm{d}}{\mathrm{d} x} \log \tau(x)=\frac{2}{y} v^{2}-\left(y+2 x+\frac{4 \theta_{0}}{y}\right) v+\left(\theta_{0}+\theta_{\infty}\right)(y+2 x) .
$$

There exist unique solutions $\Phi_{k}(z, x)$ of the equation (6.10) subject to the normalized asymptotic behavior as $z \rightarrow \infty$

$$
\Phi_{k}(z, x)=(I+O(1 / z)) e^{\frac{z^{2}+2 x z}{2} \sigma_{3}} z^{-\theta_{\infty} \sigma_{3}}, \quad z \in \Omega_{k},
$$

where $\Omega_{k}, k=1,2,3,4$, are the Stokes sectors defined in (6.2). Using (6.14), it is seen that the solutions are related to each other by the Stokes matrices

$$
\begin{gathered}
\Phi_{k+1}(z)=\Phi_{k}(z) \hat{S}_{k}, \quad k=1,2,3, \\
\Phi_{1}(z)=\Phi_{4}\left(z e^{2 \pi i}\right) \hat{S}_{4} e^{2 \pi i \theta_{\infty} \sigma_{3}},
\end{gathered}
$$

where

$$
\hat{S}_{2 k-1}=\left(\begin{array}{cc}
1 & 0 \\
\hat{s}_{2 k-1} & 1
\end{array}\right), \quad \hat{S}_{2 k}=\left(\begin{array}{cc}
1 & \hat{s}_{2 k} \\
0 & 1
\end{array}\right), \quad k=1,2 .
$$

The solution $\Phi_{1}(z, x)$ has the asymptotic behaviors as $z \rightarrow 0$

$$
\Phi_{1}(z, x)=\Phi_{1}^{(0)}(x)(I+O(z)) z^{\theta_{0} \sigma_{3}} \hat{E}
$$

with some invertible constant matrix $\hat{E}$. The monodromy data of the system (6.10) is defined as the set

$$
\left\{e^{2 \pi i \theta_{\infty}}, e^{\pi i \theta_{0}} ; \hat{S}_{1}, \hat{S}_{2}, \hat{S}_{3}, \hat{S}_{4} ; \hat{E}\right\}
$$

They satisfy the cyclic condition

$$
\hat{S}_{1} \hat{S}_{2} \hat{S}_{3} \hat{S}_{4} e^{2 \pi i \theta_{\infty} \sigma_{3}}=\hat{E}^{-1} e^{-2 \pi i \theta_{0} \sigma_{3}} \hat{E} .
$$

Thus, the Stokes multipliers $\hat{s}_{k}, k=1,2,3,4$ satisfy

$$
e^{2 \pi i \theta_{\infty}}\left(1+\hat{s}_{2} \hat{s}_{3}\right)+e^{-2 \pi i \theta_{\infty}}\left(\hat{s}_{1} \hat{s}_{4}+\left(1+\hat{s}_{1} \hat{s}_{2}\right)\left(1+\hat{s}_{3} \hat{s}_{4}\right)\right)=2 \cos \left(2 \pi \theta_{0}\right) .
$$

Moreover, the cyclic condition (6.18) specifies the connection matrices $\hat{E}$ to within a leftmultiplicative diagonal matrices; see [12]. 


\subsection{Reduction of the linear system for PIV to BHE}

In this subsection, we will derive BHE from (1.11) associated with the isomonodromy system of PIV when the PIV transcendents $y(x)$ tends to zero or infinity.

Substituting $A(z, x)$ into (1.11) gives

$$
\frac{\mathrm{d}^{2} \Phi_{1}}{\mathrm{~d} z^{2}}-\left(\frac{1}{z-\lambda}-\frac{1}{z}\right) \frac{\mathrm{d} \Phi_{1}}{\mathrm{~d} z}-Q(z, x) \Phi_{1}=0,
$$

where

$$
\begin{aligned}
Q(z, x)=z^{2}+ & 2 x z+x^{2}+2\left(1-\theta_{\infty}\right)+\frac{\theta_{0}^{2}}{z^{2}}-\frac{1}{z-\lambda}\left(z+x+\frac{\theta_{0}-v}{z}\right) \\
+ & \frac{1}{z}\left[\frac{2}{y} v^{2}-\left(y+2 x+\frac{4 \theta_{0}}{y}\right) v+\left(\theta_{0}+\theta_{\infty}\right) y+\left(1+2 \theta_{0}\right) x\right] \\
=z^{2} & +2 x z+x^{2}+2\left(1-\theta_{\infty}\right)+\frac{\theta_{0}^{2}}{z^{2}}-\frac{1}{z-\lambda}\left(z+x+\frac{\theta_{0}-v}{z}\right) \\
+ & \frac{1}{z}\left[\frac{\mathrm{d}}{\mathrm{d} x} \log \tau(x)+\left(1-2 \theta_{\infty}\right) x\right] .
\end{aligned}
$$

To derive BHE, we need to eliminate the extra singularity $\lambda=y / 2$. Thus we consider the zeros and poles of $y$. According to [23, (1.4)-(1.5)], the solution $y$ of PIV equation admits the Taylor expansion near a zero

$$
y(x)=\varepsilon(x-a)+b(x-a)^{2}+O\left((x-a)^{3}\right),
$$

the Laurent expansion near a pole

$$
y(x)=\sigma(x-a)^{-1}-a+a_{1}(x-a)+b(x-a)^{2}+O\left((x-a)^{3}\right),
$$

where $b$ is arbitrary and the parameters

$$
\varepsilon=\varepsilon_{ \pm}= \pm 4 \theta_{0}, \quad \sigma=\sigma_{ \pm}= \pm 1, \quad a_{1}=\frac{1}{3} \sigma\left(a^{2}-2+4\left(\theta_{\infty}-\sigma\right)\right) .
$$

Let us first consider the zeros of $y$. It follows from (6.11) and (6.22) that

$$
v(x)= \begin{cases}O(x-a), & \varepsilon=\varepsilon_{+}, \\ 2 \theta_{0}+O(x-a), & \varepsilon=\varepsilon_{-} .\end{cases}
$$

For $\varepsilon=\varepsilon_{+}$, taking $x \rightarrow a$ in (6.21) and then setting

$$
\Phi_{1}=z^{\theta_{0}} e^{\frac{1}{2} z^{2}+a z} w
$$

we obtain the BHE

$$
\left\{\begin{array}{l}
\frac{\mathrm{d}^{2} w}{\mathrm{~d} z^{2}}+\left(2 z+2 a+\frac{2 \theta_{0}}{z}\right) \frac{\mathrm{d} w}{\mathrm{~d} z}+\frac{p z-q}{z} w=0 \\
p=2\left(\theta_{0}+\theta_{\infty}\right), \\
q=\lim _{x \rightarrow a} \frac{\mathrm{d}}{\mathrm{d} x} \log \tau(x)-2\left(\theta_{0}+\theta_{\infty}\right) a .
\end{array}\right.
$$

For $\varepsilon=\varepsilon_{-}$, by taking $x \rightarrow a$ in (6.21) and setting

$$
\Phi_{1}=z^{\theta_{0}+1} e^{\frac{1}{2} z^{2}+a z} w
$$


we obtain the BHE

$$
\left\{\begin{array}{l}
\frac{\mathrm{d}^{2} w}{\mathrm{~d} z^{2}}+\left(2 z+2 a+\frac{2 \theta_{0}+2}{z}\right) \frac{\mathrm{d} w}{\mathrm{~d} z}+\frac{p z-q}{z} w=0, \\
p=2\left(\theta_{0}+\theta_{\infty}+1\right), \\
q=\lim _{x \rightarrow a} \frac{\mathrm{d}}{\mathrm{d} x} \log \tau(x)-2\left(\theta_{0}+\theta_{\infty}+1\right) a .
\end{array}\right.
$$

Next we focus on the poles of $y$. It is seen from (6.11) and (6.23) that

$$
v(x)= \begin{cases}\frac{1}{2}(x-a)^{-2}+O(1), & \sigma=\sigma_{+}, \\ \theta_{0}+\theta_{\infty}+O(x-a), & \sigma=\sigma_{-} .\end{cases}
$$

By taking the limit $x \rightarrow a$ in (6.21) and then making the transformation

$$
\Phi_{1}=z^{\theta_{0}} e^{\frac{1}{2} z^{2}+a z} w,
$$

we obtain another two BHEs

$$
\left\{\begin{array}{l}
\frac{\mathrm{d}^{2} w}{\mathrm{~d} z^{2}}+\left(2 z+2 a+\frac{2 \theta_{0}+1}{z}\right) \frac{\mathrm{d} w}{\mathrm{~d} z}+\frac{p z-q}{z} w=0 \\
p=2\left(\theta_{0}+\theta_{\infty}+1\right) \\
q=\lim _{x \rightarrow a}\left(\frac{\mathrm{d}}{\mathrm{d} x} \log \tau(x)-\frac{1}{x-a}\right)-\left(2 \theta_{0}+2 \theta_{\infty}+1\right) a \\
\quad=-b-\left(2 \theta_{0}+2 \theta_{\infty}-\frac{1}{2}\right) a
\end{array}\right.
$$

for $\sigma=\sigma_{+}=1$,

$$
\left\{\begin{array}{l}
\frac{\mathrm{d}^{2} w}{\mathrm{~d} z^{2}}+\left(2 z+2 a+\frac{2 \theta_{0}+1}{z}\right) \frac{\mathrm{d} w}{\mathrm{~d} z}+\frac{p z-q}{z} w=0, \\
p=2\left(\theta_{0}+\theta_{\infty}\right) \\
q=\lim _{x \rightarrow a} \frac{\mathrm{d}}{\mathrm{d} x} \log \tau(x)-2\left(\theta_{0}+\theta_{\infty}\right) a \\
=-b-\left(2 \theta_{0}+2 \theta_{\infty}+\frac{1}{2}\right) a
\end{array}\right.
$$

for $\sigma=\sigma_{-}=-1$.

\subsection{Isomonodromy set of accessory parameters of BHE}

We have shown that the BHE (1.4), with the accessory parameters $a$ and $q$, the other fixed parameters $\gamma$ and $p$ can be obtained as a limit of the first row of the isomonodromy family of $\Phi(z, x)$, when $x \rightarrow a, a$ being one of the poles or zeros of the Painlevé IV transcendents. While the accessory parameters $(a, q)$ are expressed in terms of the parameters in the Laurent or Taylor expansion of the PIV transcendents as given in (6.24)-(6.28). Therefore, we have the following description of the isomonodromy set of accessory parameters of BHE.

Theorem 5. There is a discrete set of pairs of accessory parameters $\left(a_{n}, q_{n}\right)$ such that the BHE (1.4) corresponding to these parameters has the same monodromy data described by (6.9) as the original equation with the parameters $a$ and $q$. Under the bijection given in the last equation of (6.27), this set coincides with the set of parameters $\left(a_{n}, b_{n}\right)$ in the Laurent expansion near the poles $a_{n}$ of the unique solution of PIV (6.12) with the parameter $\theta_{0}=(\gamma-1) / 2$, $\theta_{\infty}=(p-\gamma-1) / 2$ and the same monodromy data (6.9) as the BHE. 


\section{Accessory parameters of $\mathrm{CHE}$}

\subsection{Monodromy of CHE}

Consider the CHE (1.2) with the accessory parameters $a$ and $q$, the fixed parameters $\gamma, \delta$ and $p$. There exist uniquely two linear independent solutions of (1.2), namely $Y_{k}=\left(y_{k 1}, y_{k 2}\right)$, normalizing the asymptotic behavior as $z \rightarrow \infty$

$$
Y_{k}(z) \sim e^{-\frac{z}{2}} z^{-\frac{1}{2}\left(\theta_{0}+\theta_{1}\right)}(1,1 / z) e^{\frac{z}{2} \sigma_{3}} z^{-\frac{1}{2} \theta_{\infty} \sigma_{3}}, \quad z \in \Omega_{k},
$$

where the parameters $\theta_{0}=\gamma, \theta_{1}=\delta-1, \theta_{\infty}=2 p-\gamma-\delta+1$ and the Stokes sectors $\Omega_{k}, k=1,2$ are defined by

$$
\Omega_{k}=\left\{z \in \mathbb{C}: \frac{\pi}{2}(-2 k+1)<\arg z<\frac{\pi}{2}(-2 k+5)\right\} .
$$

The solutions are related to each other by the Stokes matrices

$$
\begin{gathered}
Y_{2}(z)=Y_{1}(z) S_{1}, \quad S_{1}=\left(\begin{array}{cc}
1 & 0 \\
s_{1} & 1
\end{array}\right), \\
Y_{1}\left(z e^{2 \pi i}\right) e^{\pi i\left(\theta_{0}+\theta_{1}\right)} e^{\pi i \theta_{\infty} \sigma_{3}}=Y_{2}(z) S_{2}, \quad S_{2}=\left(\begin{array}{cc}
1 & s_{2} \\
0 & 1
\end{array}\right) .
\end{gathered}
$$

Near the regular singular points, $Y_{1}$ has the asymptotic behaviors

$$
Y_{1}(z) \sim\left(z^{0}, z^{1-\theta_{0}}\right) E_{0}, \quad Y_{1}(z) \sim\left((z-a)^{0},(z-a)^{-\theta_{1}}\right) E_{1},
$$

with some invertible constant matrices $E_{0}$ and $E_{1}$. After an analytic continuation along a closed loop around a singular point, we obtain the relations

$$
Y_{1}\left(z_{k}+e^{2 \pi i}\left(z-z_{k}\right)\right)=Y_{1}(z) e^{-\pi i \theta_{k}} M_{k}, \quad M_{k}=E_{k}^{-1} e^{\pi i \theta_{k} \sigma_{3}} E_{k}
$$

for $k=0,1$, where $z_{0}=0$ and $z_{1}=a$. We define the monodromy data of the CHE equation as

$$
\left\{e^{\pi i \theta_{\infty}}, e^{\pi i \theta_{0}}, e^{\pi i \theta_{1}} ; S_{1}, S_{1} ; E_{0}, E_{1}\right\} .
$$

According to ( $(\mathbf{7 . 3})-(\underline{7.5})$, the monodromy matrices satisfies the cyclic condition

$$
M_{1} M_{0}=S_{1} S_{2} e^{-\pi i \theta_{\infty} \sigma_{3}} .
$$

Suppose

$$
2 \cos (\pi \sigma)=\operatorname{Tr} M_{1} M_{2}=2 \cos \left(\pi \theta_{\infty}\right)+s_{1} s_{2} e^{\pi i \theta_{\infty}},
$$

with $0 \leq \operatorname{Re} \sigma \leq 1$. Then, the connection matrices can be parameterized in terms of $\sigma$ and the other free parameter $s$; see [19]. For instance, we have for $\sigma \neq 0$ [19, Equation (3.7)]:

$$
\begin{gathered}
D_{1} E_{1} D=\left(\begin{array}{cc}
\sin \left(\frac{\pi}{2}\left(\theta_{1}+\theta_{0}+\sigma\right)\right) \sin \left(\frac { \pi } { 2 } \left(\theta_{1}-\theta_{0}\right.\right. & +\sigma)) \\
-s^{-1} & -s \sin \left(\frac{\pi}{2}\left(\theta_{1}+\theta_{0}-\sigma\right)\right) \sin \left(\frac{\pi}{2}\left(\theta_{1}-\theta_{0}-\sigma\right)\right) \\
1
\end{array}\right) \\
\times\left(\begin{array}{cc}
e^{-\frac{1}{2} \pi i \sigma} & \sin \left(\frac{\pi}{2}\left(\theta_{\infty}+\sigma\right)\right) \\
e^{\frac{1}{2} \pi i \sigma} & \sin \left(\frac{\pi}{2}\left(\theta_{\infty}-\sigma\right)\right)
\end{array}\right)
\end{gathered}
$$

and

$$
\begin{array}{cc}
D_{0} E_{0} D=\left(\begin{array}{cc}
\sin \left(\frac{\pi}{2}\left(\theta_{1}+\theta_{0}+\sigma\right)\right) & -s e^{-\pi i \sigma} \sin \left(\frac{\pi}{2}\left(\theta_{1}+\theta_{0}-\sigma\right)\right) \\
-s^{-1} e^{\pi i \sigma} \sin \left(\frac{\pi}{2}\left(\theta_{1}-\theta_{0}+\sigma\right)\right) & \sin \left(\frac{\pi}{2}\left(\theta_{1}-\theta_{0}-\sigma\right)\right)
\end{array}\right) \\
\times\left(\begin{array}{cc}
e^{-\frac{1}{2} \pi i \sigma} & \sin \left(\frac{\pi}{2}\left(\theta_{\infty}+\sigma\right)\right) \\
e^{\frac{1}{2} \pi i \sigma} & \sin \left(\frac{\pi}{2}\left(\theta_{\infty}-\sigma\right)\right)
\end{array}\right),
\end{array}
$$

with some invertible diagonal matrices $D_{1}, D_{0}$ and $D$. 


\subsection{Isomonodromy deformation and $\mathrm{PV}$ equation}

To study the isomonodromy deformation of the CHE, it is convenient to consider a $2 \times 2$ matrix system, which has one irregular singular point of rank 1 at infinity and two regular singularities. In [12, 20], the isomonodromy deformation of such a matrix system has been considered.

Recall the following Lax pair for PV (see [20, (C.38)-(C.39)]):

$$
\left\{\begin{array}{l}
\frac{\partial \Phi(z, x)}{\partial z}=A(z, x) \Phi(z, x), \quad A(z, x)=\frac{\sigma_{3}}{2}+\frac{A_{0}(x)}{z}+\frac{A_{1}(x)}{z-x} \\
\frac{\partial \Phi(z, x)}{\partial x}=B(z, x) \Phi(z, x), \quad B(z, x)=-\frac{A_{1}(x)}{z-x}
\end{array}\right.
$$

the coefficients

$$
\begin{aligned}
& A_{0}(x)=\left(\begin{array}{cc}
v+\frac{\theta_{0}}{2} & -u\left(v+\theta_{0}\right) \\
\frac{v}{u} & -v-\frac{\theta_{0}}{2}
\end{array}\right), \\
& A_{1}(x)=\left(\begin{array}{cc}
-v-\frac{\theta_{0}+\theta_{\infty}}{2} & y u\left(v+\frac{\theta_{0}-\theta_{1}+\theta_{\infty}}{2}\right) \\
-\frac{1}{y u}\left(v+\frac{\theta_{0}+\theta_{1}+\theta_{\infty}}{2}\right) & v+\frac{\theta_{0}+\theta_{\infty}}{2}
\end{array}\right) .
\end{aligned}
$$

The compatibility condition of the above Lax pair reads

$$
\left\{\begin{array}{l}
x \frac{\mathrm{d} y}{\mathrm{~d} x}=x y-2 v(y-1)^{2}-(y-1)\left(\frac{\theta_{0}-\theta_{1}+\theta_{\infty}}{2} y-\frac{3 \theta_{0}+\theta_{1}+\theta_{\infty}}{2}\right), \\
x \frac{\mathrm{d} v}{\mathrm{~d} x}=y v\left(v+\frac{\theta_{0}-\theta_{1}+\theta_{\infty}}{2}\right)-\frac{1}{y}\left(v+\theta_{0}\right)\left(v+\frac{\theta_{0}+\theta_{1}+\theta_{\infty}}{2}\right), \\
x \frac{\mathrm{d} u}{\mathrm{~d} x}=u\left[-2 v-\theta_{0}-\theta_{\infty}+y\left(v+\frac{\theta_{0}-\theta_{1}+\theta_{\infty}}{2}\right)+\frac{1}{y}\left(v+\frac{\theta_{0}+\theta_{1}+\theta_{\infty}}{2}\right)\right],
\end{array}\right.
$$

which implies the PV equation

$$
\frac{\mathrm{d}^{2} y}{\mathrm{~d} x^{2}}=\left(\frac{1}{2 y}+\frac{1}{y-1}\right)\left(\frac{\mathrm{d} y}{\mathrm{~d} x}\right)^{2}-\frac{1}{x} \frac{\mathrm{d} y}{\mathrm{~d} x}+\frac{(y-1)^{2}}{x^{2}}\left(\alpha y+\frac{\beta}{y}\right)+\frac{\gamma y}{x}-\frac{y(y+1)}{2(y-1)},
$$

where

$$
\alpha=\frac{1}{8}\left(\theta_{0}-\theta_{1}+\theta_{\infty}\right)^{2}, \quad \beta=-\frac{1}{8}\left(\theta_{0}-\theta_{1}-\theta_{\infty}\right)^{2}, \quad \gamma=1-\theta_{0}-\theta_{1} .
$$

There exist unique solutions $\Phi_{k}(z, x)$ of the equation (7.11), which satisfies the normalized asymptotic behavior as $z \rightarrow \infty$

$$
\Phi_{k}(z, x)=(I+O(1 / z)) e^{\frac{z}{2} \sigma_{3}} z^{-\frac{1}{2} \theta_{\infty} \sigma_{3}}, \quad z \in \Omega_{k},
$$

where $\Omega_{k}, k=1,2$, are the Stokes sectors defined in (7.2). The solutions are related to each other by the Stokes matrices

$$
\begin{gathered}
\Phi_{2}(z)=\Phi_{1}(z) \hat{S}_{1}, \quad \hat{S}_{1}=\left(\begin{array}{cc}
1 & 0 \\
\hat{s}_{1} & 1
\end{array}\right) \\
\Phi_{1}\left(z e^{2 \pi i}\right) e^{\pi i \theta_{\infty} \sigma_{3}}=\Phi_{2}(z) \hat{S}_{2}, \quad \hat{S}_{2}=\left(\begin{array}{cc}
1 & \hat{s}_{2} \\
0 & 1
\end{array}\right) .
\end{gathered}
$$

Near the regular singular points $z_{0}=0$ and $z_{1}=a, \Phi_{1}(z, x)$ has the asymptotic behaviors

$$
\Phi_{1}(z, x)=\Phi_{1}^{(k)}(x)\left(I+O\left(z-z_{k}\right)\right)\left(z-z_{k}\right)^{\frac{1}{2} \theta_{k} \sigma_{3}} \hat{E}_{k}, \quad z \rightarrow z_{k},
$$


with some invertible constant matrices $\hat{E}_{k}$ and $z$-independent matrices $\Phi_{1}^{(k)}(x), k=0,1$. The monodromy data of the system (7.11) is defined as the set

$$
\left\{e^{\pi i \theta_{\infty}}, e^{\pi i \theta_{k}} ; \hat{S}_{1}, \hat{S}_{2} ; \hat{E}_{0}, \hat{E}_{1}\right\} \text {. }
$$

They satisfy the cyclic condition

$$
\hat{M}_{1} \hat{M}_{0}=\hat{S}_{1} \hat{S}_{2} e^{-\pi i \theta_{\infty} \sigma_{3}}, \quad \hat{M}_{k}=\hat{E}_{k}^{-1} e^{\pi i \theta_{k} \sigma_{3}} \hat{E}_{k}, \quad k=0,1 .
$$

\subsection{Reduction of the linear systems for PV to CHE}

In this subsection, we will derive CHE from the linear systems (1.11) and (1.12) for PV at the poles, zeros and 1-points (that is, $y(a)=1$ ) of the solutions to PV.

Substituting the elements of $A(z, x)$ into (1.11) gives

$$
\frac{\mathrm{d}^{2} \Phi_{1}}{\mathrm{~d} z^{2}}-\left(\frac{1}{z-\lambda_{1}}-\frac{1}{z}-\frac{1}{z-x}\right) \frac{\mathrm{d} \Phi_{1}}{\mathrm{~d} z}-P(z, x) \Phi_{1}=0
$$

where

$$
\left\{\begin{aligned}
\lambda_{1}=\lambda_{1}(x) & =\frac{x}{1-\frac{y\left(v+\frac{1}{2}\left(\theta_{0}-\theta_{1}+\theta_{\infty}\right)\right)}{v+\theta_{0}}}, \\
P(z, x)= & \frac{1}{4}+\frac{\theta_{0}^{2}}{4 z^{2}}+\frac{M(x)}{z(z-x)}+\frac{\theta_{1}^{2}}{4(z-x)^{2}}+\frac{2-\theta_{\infty}}{2(z-x)} \\
& -\frac{1}{z-\lambda_{1}}\left(\frac{1}{2}+\frac{2 v+\theta_{0}}{2 z}-\frac{2 v+\theta_{0}+\theta_{\infty}}{2(z-x)}\right), \\
M(x)= & y v\left(v+\frac{\theta_{0}-\theta_{1}+\theta_{\infty}}{2}\right)+\frac{1}{y}\left(v+\theta_{0}\right)\left(v+\frac{\theta_{0}+\theta_{1}+\theta_{\infty}}{2}\right) \\
- & 2\left(v+\frac{\theta_{0}}{2}\right)\left(v+\frac{\theta_{0}+\theta_{\infty}}{2}\right)-\left(v+\frac{1+\theta_{0}}{2}\right) x-\frac{\theta_{\infty}}{2} .
\end{aligned}\right.
$$

In virtue of the definition of the PV $\tau$-function (see [20, (C.43)]):

$$
\begin{aligned}
x \frac{\mathrm{d}}{\mathrm{d} x} \log \tau(x)= & -\left[v-\frac{1}{y}\left(v+\frac{\theta_{0}+\theta_{1}+\theta_{\infty}}{2}\right)\right]\left[v+\theta_{0}-y\left(v+\frac{\theta_{0}-\theta_{1}+\theta_{\infty}}{2}\right)\right] \\
& -\left(v+\frac{\theta_{0}+\theta_{\infty}}{2}\right) x,
\end{aligned}
$$

the expression of $M(x)$ is simplified to

$$
M(x)=x \frac{\mathrm{d}}{\mathrm{d} x} \log \tau(x)+\frac{\left(\theta_{\infty}-1\right) x}{2}-\frac{\theta_{0}^{2}+\theta_{1}^{2}-\theta_{\infty}^{2}}{4}-\frac{\theta_{\infty}}{2} .
$$

Similarly, substituting the elements of $A(z, x)$ into (1.12) gives

$$
\frac{\mathrm{d}^{2} \Phi_{2}}{\mathrm{~d} z^{2}}-\left(\frac{1}{z-\lambda_{2}}-\frac{1}{z}-\frac{1}{z-x}\right) \frac{\mathrm{d} \Phi_{2}}{\mathrm{~d} z}-Q(z, x) \Phi_{2}=0
$$

where

$$
\left\{\begin{array}{l}
\lambda_{2}=\lambda_{2}(x)=\frac{x}{1-\frac{v+\frac{1}{2}\left(\theta_{0}+\theta_{1}+\theta_{\infty}\right)}{y v}}, \\
Q(z, x)=\frac{1}{4}+\frac{\theta_{0}^{2}}{4 z^{2}}+\frac{N(x)}{z(z-x)}+\frac{\theta_{1}^{2}}{4(z-x)^{2}}-\frac{2+\theta_{\infty}}{2(z-x)} \\
\quad+\frac{1}{z-\lambda_{2}}\left(\frac{1}{2}+\frac{2 v+\theta_{0}}{2 z}-\frac{2 v+\theta_{0}+\theta_{\infty}}{2(z-x)}\right), \\
N(x)=x \frac{\mathrm{d}}{\mathrm{d} x} \log \tau(x)+\frac{\left(\theta_{\infty}+1\right) x}{2}-\frac{\theta_{0}^{2}+\theta_{1}^{2}-\theta_{\infty}^{2}}{4}+\frac{\theta_{\infty}}{2} .
\end{array}\right.
$$


To eliminate the singularities $z=\lambda_{1}$ and $z=\lambda_{2}$ and obtain CHE, we need

$$
\begin{aligned}
& \lambda_{1}(x)=0, \quad \text { or } \quad \lambda_{1}(x)=x, \quad \text { or } \quad \lambda_{1}(x)=\infty, \\
& \lambda_{2}(x)=0, \quad \text { or } \lambda_{2}(x)=x, \text { or } \quad \lambda_{2}(x)=\infty \text {, }
\end{aligned}
$$

in (7.19) and (7.21), respectively. For this purpose, we consider the poles, zeros and 1-points of the solution $y$.

According to [16, (36.4)-(36.7)], the solution $y$ admits the Laurent expansion near a pole

$$
y(x)= \begin{cases}\varepsilon(x-a)^{-1}+b+O(x-a), & \alpha \neq 0, \\ a b(x-a)^{-2}+b(x-a)^{-1}+O(1), & \alpha=0,\end{cases}
$$

and the following Taylor expansions near a zero and near a 1-point

$$
\begin{gathered}
y(x)= \begin{cases}\delta(x-a)+b(x-a)^{2}+O\left((x-a)^{3}\right), & \beta \neq 0, \\
b(x-a)^{2}+O\left((x-a)^{3}\right), & \beta=0,\end{cases} \\
y(x)=1+\omega(x-a)+\left(\frac{1}{2}+\frac{\omega-1+\theta_{0}+\theta_{1}}{2 a}\right)(x-a)^{2}+O\left((x-a)^{3}\right),
\end{gathered}
$$

where $b$ is arbitrary and the parameters

$$
\left\{\begin{array}{l}
\varepsilon=\varepsilon_{ \pm}= \pm 2 a /\left(\theta_{0}-\theta_{1}+\theta_{\infty}\right) \\
\delta=\delta_{ \pm}= \pm\left(\theta_{0}-\theta_{1}-\theta_{\infty}\right) / 2 a \\
\omega=\omega_{ \pm}= \pm 1
\end{array}\right.
$$

From the first equation in the compatibility condition (7.12) and above expansions, we derive the behaviors of $v, \lambda_{1}, \lambda_{2}$ at each pole, zero or 1-point of $y$, as given in Table 1.

\begin{tabular}{|c|c|c|c|c|}
\hline Case & $y$ & $v$ & $\lambda_{1}$ & $\lambda_{2}$ \\
\hline$\varepsilon=\varepsilon_{+}$ & simple pole & 0 & 0 & $O(a)$ \\
\hline$\varepsilon=\varepsilon_{-}$ & simple pole & $-\frac{1}{2}\left(\theta_{0}-\theta_{1}+\theta_{\infty}\right)$ & $O(a)$ & $a$ \\
\hline$\alpha=0$ & double pole & 0 & 0 & $a$ \\
\hline$\delta=\delta_{+}$ & simple zero & $-\theta_{0}$ & $O(a)$ & 0 \\
\hline$\delta=\delta_{-}$ & simple zero & $-\frac{1}{2}\left(\theta_{0}+\theta_{1}+\theta_{\infty}\right)$ & $a$ & $O(a)$ \\
\hline$\beta=0$ & double zero & $-\frac{1}{2}\left(\theta_{0}+\theta_{1}+\theta_{\infty}\right)$ & $a$ & 0 \\
\hline$\omega=\omega_{+}$ & 1-point & $O(1)$ & $O(a)$ & $O(a)$ \\
\hline$\omega=\omega_{-}$ & 1-point & $a(x-a)^{-2}+O(1)$ & $\infty$ & $\infty$ \\
\hline
\end{tabular}

Table 1: Behaviors of $y, v, \lambda_{1}, \lambda_{2}$ at the critical point $x=a$

It is readily seen from Table 1 that the condition (7.22) or (7.23) is fulfilled when $a$ is a pole, zero or 1-point of $y$ with $y^{\prime}(a)=\omega_{-}$. Thus, the equation (7.19) or (7.21) is equivalent to CHE in these cases. For example, we consider the case $\varepsilon=\varepsilon_{+}$. According to Table 1, we have $v(a)=0, \lambda_{1}(a)=0$. Thus, by taking $x \rightarrow a$ in (7.19) and setting

$$
\Phi_{1}=z^{\frac{\theta_{0}}{2}}(z-a)^{\frac{\theta_{1}}{2}} e^{\frac{1}{2} z} w
$$


we obtain the CHE

$$
\left\{\begin{array}{l}
\frac{\mathrm{d}^{2} w}{\mathrm{~d} z^{2}}+\left(1+\frac{\theta_{0}}{z}+\frac{1+\theta_{1}}{z-a}\right) \frac{\mathrm{d} w}{\mathrm{~d} z}+\frac{p z-q}{z(z-a)} w=0, \\
p=\frac{1}{2}\left(\theta_{0}+\theta_{1}+\theta_{\infty}\right), \\
q=\lim _{x \rightarrow a} x \frac{\mathrm{d}}{\mathrm{d} x} \log \tau(x)+\frac{\left(\theta_{0}+\theta_{\infty}\right) a}{2}-\frac{\left(\theta_{0}+\theta_{1}\right)^{2}-\theta_{\infty}^{2}}{4} \\
\quad=\frac{a}{4}\left(\theta_{0}-\theta_{1}+\theta_{\infty}\right)-\frac{b}{4}\left(\theta_{0}-\theta_{1}+\theta_{\infty}\right)^{2}+\frac{1}{4}\left(\theta_{\infty}+1\right)\left(\theta_{0}-\theta_{1}+\theta_{\infty}\right)-\theta_{0} \theta_{1} .
\end{array}\right.
$$

Actually, we obtain six different CHEs with the characteristic exponents at the singular points shown in Table 2 and the accessory parameters given below:

$$
q=\lim _{x \rightarrow a} x \frac{\mathrm{d}}{\mathrm{d} x} \log \tau(x)+\frac{\left(\theta_{0}+\theta_{\infty}\right) a}{2}-\frac{\left(\theta_{0}+\theta_{1}\right)^{2}-\theta_{\infty}^{2}}{4},
$$

for $\varepsilon=\varepsilon_{+}, \delta=\delta_{+}$and $\delta=\delta_{-}$,

$$
q=\lim _{x \rightarrow a} x \frac{\mathrm{d}}{\mathrm{d} x} \log \tau(x)+\frac{\left(\theta_{0}+\theta_{\infty}+2\right) a}{2}-\frac{\left(\theta_{0}+\theta_{1}\right)^{2}-\theta_{\infty}^{2}}{4},
$$

for $\varepsilon=\varepsilon_{-}$,

$$
q=\lim _{x \rightarrow a}\left(x \frac{\mathrm{d}}{\mathrm{d} x} \log \tau(x)-\frac{a}{x-a}\right)+\frac{\left(\theta_{0}+\theta_{\infty}\right) a}{2}-\frac{\left(\theta_{0}+\theta_{1}\right)^{2}-\theta_{\infty}^{2}}{4}+1-\theta_{0}-\theta_{1},
$$

for $\omega=\omega_{-}$and employing $\Phi_{1}$,

$$
q=\lim _{x \rightarrow a}\left(x \frac{\mathrm{d}}{\mathrm{d} x} \log \tau(x)-\frac{a}{x-a}\right)+\frac{\left(\theta_{0}+\theta_{\infty}+2\right) a}{2}-\frac{\left(\theta_{0}+\theta_{1}\right)^{2}-\theta_{\infty}^{2}}{4}+1-\theta_{0}-\theta_{1},
$$

for $\omega=\omega_{-}$and using $\Phi_{2}$.

\begin{tabular}{|c|c|c|c|c|c|}
\hline Case & Equation & 0 & $a$ & $\infty$ & $p$ \\
\hline$\varepsilon=\varepsilon_{+}$ & $(7.19)$ & $\theta_{0}$ & $1+\theta_{1}$ & 1 & $\frac{1}{2}\left(\theta_{0}+\theta_{1}+\theta_{\infty}\right)$ \\
\hline$\varepsilon=\varepsilon_{-}$ & $(7.21)$ & $1+\theta_{0}$ & $\theta_{1}$ & 1 & $\frac{1}{2}\left(\theta_{0}+\theta_{1}+\theta_{\infty}+2\right)$ \\
\hline$\delta=\delta_{+}$ & $(7.21)$ & $\theta_{0}$ & $1+\theta_{1}$ & 1 & $\frac{1}{2}\left(\theta_{0}+\theta_{1}+\theta_{\infty}+2\right)$ \\
\hline$\delta=\delta_{-}$ & $(7.19)$ & $1+\theta_{0}$ & $\theta_{1}$ & 1 & $\frac{1}{2}\left(\theta_{0}+\theta_{1}+\theta_{\infty}\right)$ \\
\hline$\omega=\omega_{-}$ & $(7.19)$ & $1+\theta_{0}$ & $1+\theta_{1}$ & 1 & $\frac{1}{2}\left(\theta_{0}+\theta_{1}+\theta_{\infty}+2\right)$ \\
\hline$\omega=\omega_{-}$ & $(7.21)$ & $1+\theta_{0}$ & $1+\theta_{1}$ & 1 & $\frac{1}{2}\left(\theta_{0}+\theta_{1}+\theta_{\infty}+2\right)$ \\
\hline
\end{tabular}

Table 2: The characteristic exponents of CHEs obtained from equations (7.19) and (7.21)

We mention that the case $\lambda_{1}(x)=x$ has been considered in [6, 7]. According to Table 1, the condition $\lambda_{1}(x)=x$ is equivalent to the case that $y$ has simple zeros with coefficient $\delta=\delta_{-}$ or $y$ has double zeros.

\subsection{Isomonodromy set of accessory parameters of CHE}

We have shown that the CHE can be obtained as a limit of the first row or second row of the isomonodromy family of $\Phi(z, x)$ when $x \rightarrow a$, a being one of the poles, zeros or 1-points of 
the Painlevé V transcendents corresponding to the same monodromy data of CHE. While the accessory parameters $(a, q)$ are expressed in terms of the parameters in the Laurent or Taylor expansion of the PV transcendents. As a consequence, we have the following description of the isomonodromy set of accessory parameters of CHE.

Theorem 6. There is a discrete set of pairs of accessory parameters $\left(a_{n}, q_{n}\right)$ such that the CHE (1.2) corresponding to these parameters and the fixed parameters $\gamma, \delta$ and $p$, has the same monodromy data (7.6) as the original one with the parameters a and $q$. Under the relation given in the last equation of (7.25), this set coincides with the set of parameters $\left(a_{n}, b_{n}\right)$ in the Laurent expansion near the poles $a_{n}$ of the unique solution of $P V$ (7.13) with the parameters $\theta_{0}=\gamma, \theta_{1}=\delta-1, \theta_{\infty}=2 p-\gamma-\delta+1$ and corresponding to the same monodromy data (7.6) as the CHE.

\section{Accessory parameters of $\mathrm{HE}$}

\subsection{Reduction of the linear system for PVI to HE}

In this subsection, we will derive HE from the linear system (1.11) for PVI at the poles, zeros, 1-points $(y(x)=1)$ and fixed points $(y(x)=x)$ of the solutions of PVI.

Consider the following Lax pair for PVI equation (see [20, (C.46)-(C.47)]):

$$
\begin{cases}\frac{\partial \Phi(z, x)}{\partial z}=A(z, x) \Phi(z, x), & A(z, x)=\frac{A_{0}}{z}+\frac{A_{1}}{z-1}+\frac{A_{2}}{z-x} \\ \frac{\partial \Phi(z, x)}{\partial x}=B(z, x) \Phi(z, x), & B(z, x)=-\frac{A_{2}}{z-x}\end{cases}
$$

where

$$
A_{i}=\left(\begin{array}{cc}
v_{i}+\theta_{i} & -u_{i} v_{i} \\
u_{i}^{-1}\left(v_{i}+\theta_{i}\right) & -v_{i}
\end{array}\right), \quad i=0,1,2
$$

If we set

$$
\left\{\begin{array}{l}
\kappa_{1}=-\frac{1}{2}\left(\theta_{0}+\theta_{1}+\theta_{2}-\theta_{\infty}\right), \\
\kappa_{2}=-\frac{1}{2}\left(\theta_{0}+\theta_{1}+\theta_{2}+\theta_{\infty}\right), \\
A_{0}+A_{1}+A_{2}=-\left(\begin{array}{cc}
\kappa_{1} & 0 \\
0 & \kappa_{2}
\end{array}\right), \\
A(z, x)_{12}=\frac{-u_{0} v_{0}}{z}+\frac{-u_{1} v_{1}}{z-1}+\frac{-u_{2} v_{2}}{z-x}=\frac{k(z-y)}{z(z-1)(z-x)} \\
v=\frac{v_{0}+\theta_{0}}{y}+\frac{v_{1}+\theta_{1}}{y-1}+\frac{v_{2}+\theta_{2}}{y-x}
\end{array}\right.
$$

then the compatibility condition of the above Lax pair gives

$$
\left\{\begin{aligned}
\frac{\mathrm{d} y}{\mathrm{~d} x}= & \frac{y(y-1)(y-x)}{x(x-1)}\left(2 v-\frac{\theta_{0}}{y}-\frac{\theta_{1}}{y-1}-\frac{\theta_{2}-1}{y-x}\right) \\
\frac{\mathrm{d} v}{\mathrm{~d} x}= & \frac{1}{x(x-1)}\left\{\left[-3 y^{2}+2(1+x) y-x\right] v^{2}+\left[(2 y-1-x) \theta_{0}+(2 y-x) \theta_{1}\right.\right. \\
& \left.\left.+(2 y-1)\left(\theta_{2}-1\right)\right] v-\kappa_{1}\left(\kappa_{2}+1\right)\right\} \\
\frac{\mathrm{d} k}{\mathrm{~d} x}= & k\left(\theta_{\infty}-1\right) \frac{y-x}{x(x-1)}
\end{aligned}\right.
$$


which leads to the PVI equation

$$
\begin{aligned}
\frac{\mathrm{d}^{2} y}{\mathrm{~d} x^{2}}= & \frac{1}{2}\left(\frac{1}{y}+\frac{1}{y-1}+\frac{1}{y-x}\right)\left(\frac{\mathrm{d} y}{\mathrm{~d} x}\right)^{2}-\left(\frac{1}{x}+\frac{1}{x-1}+\frac{1}{y-x}\right) \frac{\mathrm{d} y}{\mathrm{~d} x} \\
& +\frac{y(y-1)(y-x)}{x^{2}(x-1)^{2}}\left(\alpha_{0}+\beta_{0} \frac{x}{y^{2}}+\gamma_{0} \frac{x-1}{(y-1)^{2}}+\delta_{0} \frac{x(x-1)}{(y-x)^{2}}\right),
\end{aligned}
$$

where

$$
\alpha_{0}=\frac{1}{2}\left(\theta_{\infty}-1\right)^{2}, \quad \beta_{0}=-\frac{1}{2} \theta_{0}^{2}, \quad \gamma_{0}=\frac{1}{2} \theta_{1}^{2}, \quad \delta_{0}=\frac{1}{2}\left(1-\theta_{2}^{2}\right) .
$$

The PVI $\tau$-function is defined by (see [20, (C.57)]):

$$
\begin{aligned}
x(x-1) \frac{\mathrm{d}}{\mathrm{d} x} \log \tau(x)= & y(y-1)(y-x)\left\{v^{2}-\left(\frac{\theta_{0}}{y}+\frac{\theta_{1}}{y-1}+\frac{\theta_{2}}{y-x}\right) v+\frac{\kappa_{1} \kappa_{2}}{y(y-1)}\right\} \\
& +\theta_{0} \theta_{2}(x-1)+\theta_{1} \theta_{2} x .
\end{aligned}
$$

Substituting the entries of $A(z, x)$ into (1.11) gives

$$
\frac{\mathrm{d}^{2} \Phi_{1}}{\mathrm{~d} z^{2}}+P(z, x) \frac{\mathrm{d} \Phi_{1}}{\mathrm{~d} z}+Q(z, x) \Phi_{1}=0
$$

where

$$
\left\{\begin{array}{l}
P(z, x)=\frac{1-\theta_{0}}{z}+\frac{1-\theta_{1}}{z-1}+\frac{1-\theta_{2}}{z-x}-\frac{1}{z-y}, \\
Q(z, x)=\frac{\kappa_{1}\left(\kappa_{2}+1\right)}{z(z-1)}-\frac{M(x)}{z(z-1)(z-x)}+\frac{y(y-1) v}{z(z-1)(z-y)}, \\
M(x)=x(x-1) \frac{\mathrm{d}}{\mathrm{d} x} \log \tau(x)+\kappa_{1}(y-x)+y(y-1) v-\theta_{0} \theta_{2}(x-1)-\theta_{1} \theta_{2} x .
\end{array}\right.
$$

It is clear that the extra singularity $z=y$ can be removed by considering the critical values

$$
y(a)=0, \text { or } y(a)=1, \text { or } y(a)=a, \text { or } y(a)=\infty .
$$

First, we consider the critical value $y(a)=a$. By taking the limit $x \rightarrow a$ in (8.6), we obtain the following HE directly

$$
\left\{\begin{array}{l}
\frac{\mathrm{d}^{2} \Phi_{1}}{\mathrm{~d} z^{2}}+\left(\frac{1-\theta_{0}}{z}+\frac{1-\theta_{1}}{z-1}+\frac{-\theta_{2}}{z-a}\right) \frac{\mathrm{d} \Phi_{1}}{\mathrm{~d} z}+\frac{p z-q}{z(z-1)(z-a)} \Phi_{1}=0, \\
p=\frac{1}{4}\left(\theta_{0}+\theta_{1}+\theta_{2}-\theta_{\infty}\right)\left(\theta_{0}+\theta_{1}+\theta_{2}+\theta_{\infty}-2\right), \\
q=\lim _{x \rightarrow a} x(x-1) \frac{\mathrm{d}}{\mathrm{d} x} \log \tau(x)+\kappa_{1}\left(\kappa_{2}+1\right) a-\theta_{0} \theta_{2}(a-1)-\theta_{1} \theta_{2} a .
\end{array}\right.
$$

The equation (8.7) has also been obtained in 1, 3, 8. It was also noted therein that the condition $y(a)=a$ can be expressed in terms of the associated $\tau$-function.

Next, we consider the poles of $y$. Let $x=a(a \neq 0,1)$ be a movable pole of $y(x)$. Then $y(x)$ possesses the following Laurent expansions (see [16, (46.7)]):

$$
y(x)= \begin{cases}\frac{\varepsilon}{x-a}+b+O(x-a), & \alpha_{0} \neq 0, \\ \frac{b}{(x-a)^{2}}+\frac{(2 a-1) b}{a(a-1)(x-a)}+c_{0}+O(x-a), & \alpha_{0}=0,\end{cases}
$$

where $\varepsilon=\varepsilon_{ \pm}= \pm a(a-1) /\left(\theta_{\infty}-1\right), b$ is arbitrary and

$$
c_{0}=\frac{1}{3}(a+1)+\frac{b}{12 a^{2}(a-1)^{2}}\left\{12 a(a-1)+1-a \theta_{0}^{2}+(a-1) \theta_{1}^{2}-a(a-1)\left(\theta_{2}^{2}-1\right)\right\} .
$$

We derive HE by considering each case separately. 
Case 1: $a$ is a simple pole with residue $\varepsilon=\varepsilon_{+}$and $\theta_{\infty} \neq 0$

In this case, we have from (8.5), (8.6) and (8.8) that

$$
\begin{aligned}
M(x)= & x(x-1) \frac{\mathrm{d}}{\mathrm{d} x} \log \tau(x)+\left(\theta_{\infty}-1\right) b-\kappa_{1} a-\theta_{0} \theta_{2}(a-1)-\theta_{1} \theta_{2} a \\
& +\frac{1}{2}\left[-\theta_{0}+\left(\theta_{2}-1\right)(a-1)-2 a+1-\left(\theta_{\infty}-1\right) a\right]+O(x-a),
\end{aligned}
$$

and

$$
\begin{aligned}
x(x-1) & \frac{\mathrm{d}}{\mathrm{d} x} \log \tau(x)=\theta_{\infty}\left(1-\theta_{\infty}\right) b \\
& -\frac{1}{2} \theta_{\infty}\left[-\theta_{0}+\left(\theta_{2}-1\right)(a-1)-2 a+1-\left(\theta_{\infty}-1\right) a\right]-\kappa_{1}^{2}(a-1) \\
& -\kappa_{1} \kappa_{2} a-\kappa_{1}\left[a \theta_{0}+(a-1) \theta_{1}\right]+\theta_{0} \theta_{2}(a-1)+\theta_{1} \theta_{2} a+O(x-a) .
\end{aligned}
$$

This, together with $\theta_{\infty} \neq 0$, implies that

$$
\begin{aligned}
M(x)= & \left(1-\theta_{\infty}^{-1}\right) x(x-1) \frac{\mathrm{d}}{\mathrm{d} x} \log \tau(x)-\kappa_{1} a-\theta_{\infty}^{-1}\left\{\kappa_{1}^{2}(a-1)+\kappa_{1} \kappa_{2} a+\kappa_{1}\left[a \theta_{0}\right.\right. \\
& \left.\left.+(a-1) \theta_{1}\right]\right\}-\left(1-\theta_{\infty}^{-1}\right)\left[\theta_{0} \theta_{2}(a-1)+\theta_{1} \theta_{2} a\right]+O(x-a) .
\end{aligned}
$$

Taking $x \rightarrow a$ in (8.6), we obtain the HE

$$
\left\{\begin{aligned}
& \frac{\mathrm{d}^{2} \Phi_{1}}{\mathrm{~d} z^{2}}+\left(\frac{1-\theta_{0}}{z}+\frac{1-\theta_{1}}{z-1}+\frac{1-\theta_{2}}{z-a}\right) \frac{\mathrm{d} \Phi_{1}}{\mathrm{~d} z}+\frac{p z-q}{z(z-1)(z-a)} \Phi_{1}=0 \\
& p= \frac{1}{4}\left(\theta_{0}+\theta_{1}+\theta_{2}-\theta_{\infty}\right)\left(\theta_{0}+\theta_{1}+\theta_{2}+\theta_{\infty}-4\right) \\
& q= \lim _{x \rightarrow a}\left(1-\theta_{\infty}^{-1}\right) x(x-1) \frac{\mathrm{d}}{\mathrm{d} x} \log \tau(x)+\kappa_{1}\left(\kappa_{2}+2\right) a-\theta_{\infty}^{-1}\left\{\kappa_{1}^{2}(a-1)\right. \\
&\left.\quad+\kappa_{1} \kappa_{2} a+\kappa_{1}\left[a \theta_{0}+(a-1) \theta_{1}\right]\right\}-\left(1-\theta_{\infty}^{-1}\right)\left[\theta_{0} \theta_{2}(a-1)+\theta_{1} \theta_{2} a\right]
\end{aligned}\right.
$$

where $\kappa_{1}$ and $\kappa_{2}$ are given in (8.2).

Case 2: $a$ is a simple pole with residue $\varepsilon=\varepsilon_{+}$and $\theta_{\infty}=0$

When $\theta_{\infty}=0$, it is seen from (8.9) and (8.10) that

$$
\begin{aligned}
M(x)= & x(x-1) \frac{\mathrm{d}}{\mathrm{d} x} \log \tau(x)-b+\frac{1}{2}\left[-\theta_{0}+\left(\theta_{2}-1\right)(a-1)-a\right]-\kappa_{1} a \\
& -\theta_{0} \theta_{2}(a-1)-\theta_{1} \theta_{2} a+O(x-a),
\end{aligned}
$$

and

$$
\begin{aligned}
x(x-1) \frac{\mathrm{d}}{\mathrm{d} x} \log \tau(x)= & -\kappa_{1}^{2}(a-1)-\kappa_{1} \kappa_{2} a-\kappa_{1}\left[a \theta_{0}+(a-1) \theta_{1}\right] \\
& +\theta_{0} \theta_{2}(a-1)+\theta_{1} \theta_{2} a+O(x-a) .
\end{aligned}
$$

It is straightforward that the free parameter $b$ is independent of $\tau(x)$. To express the accessory parameter in terms of the $\tau$-function as (8.11), we need the Schlesinger transformation used by 
Dubrovin and Kapaev to shift the formal monodromy at infinity $\theta_{\infty}$ by -2 , i.e. $\theta_{\infty} \mapsto \theta_{\infty}-2$; see [10, Sec. 2.3.2]. As a consequence, we obtain the HE

$$
\left\{\begin{array}{l}
\frac{\mathrm{d}^{2} \Phi_{1}}{\mathrm{~d} z^{2}}+\left(\frac{1-\theta_{0}}{z}+\frac{1-\theta_{1}}{z-1}+\frac{1-\theta_{2}}{z-a}\right) \frac{\mathrm{d} \Phi_{1}}{\mathrm{~d} z}+\frac{p z-q}{z(z-1)(z-a)} \Phi_{1}=0 \\
p=\frac{1}{4}\left(\theta_{0}+\theta_{1}+\theta_{2}+2\right)\left(\theta_{0}+\theta_{1}+\theta_{2}-6\right) \\
q=\lim _{x \rightarrow a} \frac{3}{2} x(x-1) \frac{\mathrm{d}}{\mathrm{d} x} \log \tilde{\tau}(x)+\tilde{\kappa}_{1}\left(\tilde{\kappa}_{2}+2\right) a+\frac{1}{2}\left\{\tilde{\kappa}_{1}^{2}(a-1)\right. \\
\left.\quad+\tilde{\kappa}_{1} \tilde{\kappa}_{2} a+\tilde{\kappa}_{1}\left[a \theta_{0}+(a-1) \theta_{1}\right]\right\}-\frac{3}{2}\left[\theta_{0} \theta_{2}(a-1)+\theta_{1} \theta_{2} a\right]
\end{array}\right.
$$

where $\tilde{\tau}(x)$ is a new $\tau$-function defined by

$$
\begin{aligned}
x(x-1) \frac{\mathrm{d}}{\mathrm{d} x} \log \tilde{\tau}(x)= & y(y-1)(y-x)\left\{v^{2}-\left(\frac{\theta_{0}}{y}+\frac{\theta_{1}}{y-1}+\frac{\theta_{2}}{y-x}\right) v+\frac{\tilde{\kappa}_{1} \tilde{\kappa}_{2}}{y(y-1)}\right\} \\
& +\theta_{0} \theta_{2}(x-1)+\theta_{1} \theta_{2} x
\end{aligned}
$$

with

$$
\left\{\begin{array}{l}
\tilde{\kappa}_{1}=-\frac{1}{2}\left(\theta_{0}+\theta_{1}+\theta_{2}+2\right), \\
\tilde{\kappa}_{2}=-\frac{1}{2}\left(\theta_{0}+\theta_{1}+\theta_{2}-2\right) .
\end{array}\right.
$$

Case 3: $a$ is a simple pole with residue $\varepsilon=\varepsilon_{-}$

From (8.6) and (8.8), it follows that

$$
\begin{aligned}
M(x)= & x(x-1) \frac{\mathrm{d}}{\mathrm{d} x} \log \tau(x)-\frac{a(a-1)}{x-a}+\frac{1}{2}\left(\theta_{0}+\theta_{1}+2 \theta_{2}-4\right) a \\
& +1-\frac{1}{2}\left(\theta_{0}+\theta_{2}\right)-\theta_{0} \theta_{2}(a-1)-\theta_{1} \theta_{2} a+O(x-a) .
\end{aligned}
$$

By taking $x \rightarrow a$ in (8.6), we arrive at the HE

$$
\left\{\begin{aligned}
\frac{\mathrm{d}^{2} \Phi_{1}}{\mathrm{~d} z^{2}}+\left(\frac{1-\theta_{0}}{z}+\frac{1-\theta_{1}}{z-1}+\frac{1-\theta_{2}}{z-a}\right) \frac{\mathrm{d} \Phi_{1}}{\mathrm{~d} z}+\frac{p z-q}{z(z-1)(z-a)} \Phi_{1}=0, \\
p=\frac{1}{4}\left(\theta_{0}+\theta_{1}+\theta_{2}-\theta_{\infty}-2\right)\left(\theta_{0}+\theta_{1}+\theta_{2}+\theta_{\infty}-2\right), \\
q=\lim _{x \rightarrow a}\left(x(x-1) \frac{\mathrm{d}}{\mathrm{d} x} \log \tau(x)-\frac{a(a-1)}{x-a}\right)+\left(\kappa_{1}+1\right)\left(\kappa_{2}+1\right) a \\
\quad+\frac{1}{2}\left(\theta_{0}+\theta_{1}+2 \theta_{2}-4\right) a+1-\frac{1}{2}\left(\theta_{0}+\theta_{2}\right)-\theta_{0} \theta_{2}(a-1)-\theta_{1} \theta_{2} a \\
=-\left(1-\theta_{\infty}\right)^{2} b-\frac{1}{4}\left(2\left(\theta_{0}+\theta_{1}\right)-\left(\theta_{0}+\theta_{1}\right)^{2}+\theta_{2}^{2}-\left(\theta_{\infty}-2\right)^{2}+4\left(\theta_{\infty}-1\right)\right) a \\
\quad-\frac{1}{2}\left(\theta_{0}+\theta_{2}\right)+\theta_{\infty}-1+\frac{1}{4}\left(\left(\theta_{0}+\theta_{2}\right)^{2}-\theta_{1}^{2}+\left(\theta_{\infty}-2\right)^{2}\right)
\end{aligned}\right.
$$

where $\kappa_{1}$ and $\kappa_{2}$ are given in (8.2). 


\section{Case 4: $a$ is a double pole}

We see from (8.6) and (8.8) that

$$
\begin{aligned}
M(x)= & x(x-1) \frac{\mathrm{d}}{\mathrm{d} x} \log \tau(x)-\frac{a(a-1)}{x-a}-\frac{1}{2}\left(\theta_{0}+\theta_{1}-4\right) a-\frac{1}{2}\left(\theta_{0}+\theta_{2}\right) \\
& -\theta_{0} \theta_{2}(a-1)-\theta_{1} \theta_{2} a+O(x-a) .
\end{aligned}
$$

By taking $x \rightarrow a$ in (8.6), we arrive at the $\mathrm{HE}$

$$
\left\{\begin{array}{l}
\frac{\mathrm{d}^{2} \Phi_{1}}{\mathrm{~d} z^{2}}+\left(\frac{1-\theta_{0}}{z}+\frac{1-\theta_{1}}{z-1}+\frac{1-\theta_{2}}{z-a}\right) \frac{\mathrm{d} \Phi_{1}}{\mathrm{~d} z}+\frac{p z-q}{z(z-1)(z-a)} \Phi_{1}=0 \\
p=\frac{1}{4}\left(\theta_{0}+\theta_{1}+\theta_{2}-1\right)\left(\theta_{0}+\theta_{1}+\theta_{2}-3\right), \\
q=\lim _{x \rightarrow a}\left(x(x-1) \frac{\mathrm{d}}{\mathrm{d} x} \log \tau(x)-\frac{a(a-1)}{x-a}\right)+\kappa_{1}\left(\kappa_{2}+2\right) a \\
\quad-\frac{1}{2}\left(\theta_{0}+\theta_{1}-4\right) a-\frac{1}{2}\left(\theta_{0}+\theta_{2}\right)-\theta_{0} \theta_{2}(a-1)-\theta_{1} \theta_{2} a \\
=\frac{a^{2}(a-1)^{2}}{b}+\left(\kappa_{1}\left(\kappa_{2}+\theta_{2}+3\right)+3\right) a+\left(\kappa_{1}\left(\kappa_{2}+\theta_{1}+1\right)-1\right),
\end{array}\right.
$$

where $\kappa_{1}$ and $\kappa_{2}$ are given in (8.2), with $\theta_{\infty}=1$ in this case.

It is noted that the above HEs are also obtained from the linear system at the poles of the solutions of PVI in [10, while the accessory parameters in these equations are expressed in terms of the free parameter of the Laurent expansion of $y$ at the poles.

Finally, we consider the critical values $y(x)=0$ and $y(x)=1$. It follows from [16, (46.8)(46.9)] that $y$ admits the following Taylor expansions:

$$
\begin{gathered}
y(x)= \begin{cases}\lambda(x-a)+b(x-a)^{2}+O\left((x-a)^{3}\right), & \beta \neq 0, \\
b(x-a)^{2}+O\left((x-a)^{3}\right), & \beta=0,\end{cases} \\
y(x)= \begin{cases}1+\omega(x-a)+b(x-a)^{2}+O\left((x-a)^{3}\right), & \gamma \neq 0, \\
1+b(x-a)^{2}+O\left((x-a)^{3}\right), & \gamma=0,\end{cases}
\end{gathered}
$$

where $b$ is arbitrary and

$$
\left\{\begin{array}{l}
\lambda=\lambda_{ \pm}= \pm \frac{\theta_{0}}{a-1} \\
\omega=\omega_{ \pm}= \pm \frac{\theta_{1}}{a}
\end{array}\right.
$$

It is seen from (8.6), (8.14) and (8.15) that

$$
M(x)=x(x-1) \frac{\mathrm{d}}{\mathrm{d} x} \log \tau(x)-\theta_{0}-\kappa_{1} a-\theta_{0} \theta_{2}(a-1)-\theta_{1} \theta_{2} a+O(x-a),
$$

for $\lambda=\lambda_{+}$,

$$
M(x)=x(x-1) \frac{\mathrm{d}}{\mathrm{d} x} \log \tau(x)-\kappa_{1} a-\theta_{0} \theta_{2}(a-1)-\theta_{1} \theta_{2} a+O(x-a),
$$

for $\lambda=\lambda_{-}$,

$$
M(x)=x(x-1) \frac{\mathrm{d}}{\mathrm{d} x} \log \tau(x)-\frac{\theta_{0}}{2}-\kappa_{1} a-\theta_{0} \theta_{2}(a-1)-\theta_{1} \theta_{2} a+O(x-a),
$$


for $\beta=0$,

$$
M(x)=x(x-1) \frac{\mathrm{d}}{\mathrm{d} x} \log \tau(x)+\kappa_{1}(1-a)-\theta_{0} \theta_{2}(a-1)-\theta_{1} \theta_{2} a+O(x-a),
$$

for $\omega=\omega_{+}$,

$$
M(x)=x(x-1) \frac{\mathrm{d}}{\mathrm{d} x} \log \tau(x)+\theta_{1}+\kappa_{1}(1-a)-\theta_{0} \theta_{2}(a-1)-\theta_{1} \theta_{2} a+O(x-a),
$$

for $\omega=\omega_{-}$,

$$
M(x)=x(x-1) \frac{\mathrm{d}}{\mathrm{d} x} \log \tau(x)+\frac{\theta_{1}}{2}+\kappa_{1}(1-a)-\theta_{0} \theta_{2}(a-1)-\theta_{1} \theta_{2} a+O(x-a),
$$

for $\gamma=0$.

Taking $x \rightarrow a$ in (8.6), we then obtain six HEs. We show the exponent parameters at the singularities $0,1, a$ and $\infty$ in Table 3 and list the accessory parameters below:

$$
q=\lim _{x \rightarrow a} x(x-1) \frac{\mathrm{d}}{\mathrm{d} x} \log \tau(x)+\theta_{0}\left(\theta_{2}-1\right)+\left(\kappa_{1} \kappa_{2}+\theta_{0}-\theta_{0} \theta_{1}-\theta_{1} \theta_{2}-2 \theta_{0} \theta_{2}\right) a,
$$

for $\lambda=\lambda_{+}$,

$$
q=\lim _{x \rightarrow a} x(x-1) \frac{\mathrm{d}}{\mathrm{d} x} \log \tau(x)+\theta_{0} \theta_{2}+\left(\kappa_{1} \kappa_{2}-\theta_{0} \theta_{2}-\theta_{1} \theta_{2}\right) a,
$$

for $\lambda=\lambda_{-}$,

$$
q=\lim _{x \rightarrow a} x(x-1) \frac{\mathrm{d}}{\mathrm{d} x} \log \tau(x)+\left(\kappa_{1} \kappa_{2}-\theta_{1} \theta_{2}\right) a,
$$

for $\beta=0$,

$$
q=\lim _{x \rightarrow a} x(x-1) \frac{\mathrm{d}}{\mathrm{d} x} \log \tau(x)+\kappa_{1}+\theta_{0} \theta_{2}+\left(\kappa_{1} \kappa_{2}-\theta_{0} \theta_{2}-\theta_{1} \theta_{2}\right) a,
$$

for $\omega=\omega_{+}$,

$$
q=\lim _{x \rightarrow a} x(x-1) \frac{\mathrm{d}}{\mathrm{d} x} \log \tau(x)+\theta_{1}+\theta_{0} \theta_{2}+\kappa_{1}+\left(\kappa_{1} \kappa_{2}+\theta_{1}-\theta_{0} \theta_{1}-\theta_{0} \theta_{2}-\theta_{1} \theta_{2}\right) a,
$$

for $\omega=\omega_{-}$,

$$
q=\lim _{x \rightarrow a} x(x-1) \frac{\mathrm{d}}{\mathrm{d} x} \log \tau(x)+\kappa_{1}+\theta_{0} \theta_{2}+\left(\kappa_{1} \kappa_{2}-\theta_{0} \theta_{2}\right) a,
$$

for $\gamma=0$.

\begin{tabular}{|c|c|c|c|c|}
\hline Case & 0 & 1 & $a$ & $\infty$ \\
\hline$\lambda=\lambda_{+}$ & $\theta_{0}$ & $1-\theta_{1}$ & $1-\theta_{2}$ & $\frac{1}{4}\left(\theta_{0}-\theta_{1}-\theta_{2}+\theta_{\infty}\right)\left(\theta_{0}-\theta_{1}-\theta_{2}-\theta_{\infty}+2\right)$ \\
\hline$\lambda=\lambda_{-}$ & $-\theta_{0}$ & $1-\theta_{1}$ & $1-\theta_{2}$ & $\frac{1}{4}\left(\theta_{0}+\theta_{1}+\theta_{2}-\theta_{\infty}\right)\left(\theta_{0}+\theta_{1}+\theta_{2}+\theta_{\infty}-2\right)$ \\
\hline$\beta=0$ & 0 & $1-\theta_{1}$ & $1-\theta_{2}$ & $\frac{1}{4}\left(\theta_{1}+\theta_{2}-\theta_{\infty}\right)\left(\theta_{1}+\theta_{2}+\theta_{\infty}-2\right)$ \\
\hline$\omega=\omega_{+}$ & $1-\theta_{0}$ & $-\theta_{1}$ & $1-\theta_{2}$ & $\frac{1}{4}\left(\theta_{0}+\theta_{1}+\theta_{2}-\theta_{\infty}\right)\left(\theta_{0}+\theta_{1}+\theta_{2}+\theta_{\infty}-2\right)$ \\
\hline$\omega=\omega_{-}$ & $1-\theta_{0}$ & $\theta_{1}$ & $1-\theta_{2}$ & $\frac{1}{4}\left(\theta_{1}-\theta_{0}-\theta_{2}+\theta_{\infty}\right)\left(\theta_{1}-\theta_{0}-\theta_{2}-\theta_{\infty}+2\right)$ \\
\hline$\gamma=0$ & $1-\theta_{0}$ & 0 & $1-\theta_{2}$ & $\frac{1}{4}\left(\theta_{0}+\theta_{2}-\theta_{\infty}\right)\left(\theta_{0}+\theta_{2}+\theta_{\infty}-2\right)$ \\
\hline
\end{tabular}

Table 3: The characteristic exponents at the singularities of HEs

We mentioned that, in the cases $\lambda=\lambda_{+}$and $\omega=\omega_{-}$, use has be made of the transformations $\Phi_{1}=z^{\theta_{0}} w$ and $\Phi_{1}=(z-1)^{\theta_{1}} w$ to get the canonical form of HE (1.1). 


\subsection{Isomonodromy set of accessory parameters of $\mathrm{HE}$}

Consider the HE (1.1) with parameters $a$ and $q$. There exist uniquely two linear independent solutions of (1.1), namely $\left(y_{1}, y_{2}\right)$, satisfied the normalized asymptotic behavior as $z \rightarrow \infty$ :

$$
\left(y_{1}(z), y_{2}(z)\right) \sim\left((1 / z)^{\alpha},(1 / z)^{\beta}\right),
$$

where $\alpha$ and $\beta$ are the characteristic exponents at $z=\infty$. We have the asymptotic behaviors of $\left(y_{1}, y_{2}\right)$ near the singular points $z_{0}=0, z_{1}=1, z_{2}=a$

$$
\left(y_{1}(z), y_{2}(z)\right) \sim\left(\left(z-z_{k}\right)^{\theta_{k}},\left(z-z_{k}\right)^{0}\right) E_{k}, \quad k=0,1,2,
$$

with the characteristic exponents $\theta_{0}=1-\gamma, \theta_{1}=1-\delta, \theta_{2}=1-\epsilon$ and some invertible constant matrix $E_{k}, k=0,1,2$. Under an analytic continuation along a closed loop around a singular point, we obtain another two linear independent solutions of the same equation, which are therefore related to $\left(y_{1}, y_{2}\right)$ by

$$
\begin{gathered}
\left(y_{1}\left(z_{k}+e^{2 \pi i}\left(z-z_{k}\right)\right), y_{2}\left(z_{k}+e^{2 \pi i}\left(z-z_{k}\right)\right)\right)=\left(y_{1}(z), y_{2}(z)\right) e^{\pi i \theta_{k}} M_{k}, \\
\left(y_{1}\left(e^{2 \pi i} z\right), y_{2}\left(e^{2 \pi i} z\right)\right)=\left(y_{1}(z), y_{2}(z)\right) e^{-\pi i(\alpha+\beta)} M_{\infty} .
\end{gathered}
$$

Here the constant matrices are known as the monodromy matrices and determined by the connection matrices and the characteristic exponents

$$
M_{k}=E_{k}^{-1} e^{\pi i \theta_{k} \sigma_{3}} E_{k}, \quad M_{\infty}=e^{\pi i \theta_{\infty} \sigma_{3}},
$$

with $k=0,1,2$ and $\theta_{\infty}=-\alpha+\beta$. The monodromy data of HE (1.1) is then constituted by

$$
\left\{e^{\pi i \theta_{\infty}}, e^{\pi i \theta_{0}}, e^{\pi i \theta_{1}}, e^{\pi i \theta_{2}} ; E_{0}, E_{1}, E_{2}\right\}
$$

where the characteristic exponents are related to the fixed parameters in (1.1) by $\theta_{0}=1-\gamma$, $\theta_{1}=1-\delta, \theta_{2}=1-\epsilon$ and $\theta_{\infty}=-\alpha+\beta$. The monodromy matrices satisfies the cyclic condition

$$
M_{\infty} M_{2} M_{1} M_{0}=I .
$$

According to [19], the monodromy matrices can be written explicitly in terms of the characteristic exponents and the parameters

$$
2 \cos \left(\pi \sigma_{j k}\right)=\operatorname{Tr} M_{j} M_{k}=\operatorname{Tr} M_{k} M_{j}
$$

with $j, k=0,1,2$ and $j<k$. Moreover, by the cyclic condition, only two of the parameters $\left\{\sigma_{01}, \sigma_{02}, \sigma_{12}\right\}$ are independent. Therefore, we get the same number of independent parameters of the monodromy matrices as the parameters $a$ and $q$ in HE equation.

To study the isomonodromy deformation of the HE equation, it is convenient to consider the matrix Fuchsian system with four regular singular points. As shown in Section 8.1, the isomonodromy deformation of (8.1) is described by the PVI equation (8.3). Moreover, let $\Phi(z, x)$ be the fundamental solution of (8.1) normalized at infinity, then $\Phi(z, x)$ has the asymptotic behaviors near the singular points:

$$
\Phi(z, x)=\Phi_{0}^{(k)}(x)\left(I+O\left(z-z_{k}\right)\right)\left(z-z_{k}\right)^{\frac{1}{2} \theta_{k}}\left(z-z_{k}\right)^{\frac{1}{2} \theta_{k} \sigma_{3}} \hat{E}_{k},
$$

and

$$
\Phi(z, x)=(I+O(1 / z)) z^{\frac{\alpha+\beta}{2}} z^{-\frac{1}{2} \theta_{\infty} \sigma_{3}},
$$


with $z_{0}=0, z_{1}=1, z_{2}=x$. Here the connection matrix $\hat{E}_{k}$ are certain invertible constant matrices. Similarly, the analytic continuation of $\Phi(z, x)$ along a closed loop around the singular points are related to $\Phi(z, x)$ by the monodromy matrices

$$
\hat{M}_{\infty}=e^{\pi i \theta_{\infty} \sigma_{3}}, \quad \hat{M}_{k}=\hat{E}_{k}^{-1} e^{\pi i \theta_{k} \sigma_{3}} \hat{E}_{k},
$$

with $k=0,1,2$. While the HE can be obtained from a family of isomonodromy deformation system by taking certain limit procedure at the poles of the solution of PVI equation; see for instance (8.12). In this way, we obtain a family of accessory parameters sharing the same monodromy data as stated in the following theorem.

Theorem 7. There is a discrete set of pairs of accessory parameters $\left(a_{n}, q_{n}\right)$ such that the HE (1.1) corresponding to these parameters has the same monodromy data (8.17) as the original one with the parameters $a$ and $q$. Under the bijection given in the last equation of (8.12), and that of (8.13), this set coincides with the set of parameters $\left(a_{n}, b_{n}\right)$ in the Laurent expansion (8.8) near the poles $a_{n}$ of the unique solution of PVI equation (8.4) with the same monodromy data (8.17) as the HE.

Proof. Consider the HE (1.1) with given accessory parameters $a$ and $q$. There exist unique fundamental solutions $\left(y_{1}, y_{2}\right)$ to this equation with the normalized behavior at infinity (8.16). The solutions are corresponding to the monodromy data (8.17) as mentioned at the beginning of this section. On the other side, there is a unique solution $\Phi(z, x)$ normalized at infinity of the system (8.1) with the parameters related to the fixed parameters of HE (1.1) by $\theta_{0}=1-\gamma$, $\theta_{1}=1-\delta, \theta_{2}=1-\epsilon$ and $\theta_{\infty}=-\alpha+\beta$. Here the $x$-dependence of $\Phi(z, x)$ is described by the unique solution of the PVI equation with the Laurent expansion as given in (8.8) with the parameter $b$ given by the last equation of (8.12) and the parameter $\theta_{\infty} \neq 1$ therein. When $\theta_{\infty}=1$, we consider the Laurent expansion with a double pole at $a$ in (8.8). The solution $\Phi(z, x)$ is corresponding to the monodromy data of the form $\left\{e^{\pi i \theta_{\infty}}, e^{\pi i \theta_{0}}, e^{\pi i \theta_{1}}, e^{\pi i \theta_{2}} ; \hat{E}_{0}, \hat{E}_{1}, \hat{E}_{2}\right\}$ which is independent of $x$.

Using the limiting procedure shown in (8.12), we obtain the HE (1.1) with accessory parameters $a$ and $q$ as limit of the first row of the isomonodromy family $\Phi(z ; x)$ as $x \rightarrow a$. It follows from the $x$-independent of the monodromy data that $E_{k}=\hat{E}_{k}, k=0,1,2$. Thus, we have shown that any given accessory parameters $(a, q)$ is related by the last equation of (8.12) to the pole parameters $(a, b)$ of the unique solution of PVI corresponding to the same monodromy data (8.17) when the parameter $\theta_{\infty} \neq 1$. In the case $\theta_{\infty}=1$, we consider (8.13) and similar analysis applies. By the meromorphic property of the PVI solution, the poles of each PVI solution are discrete and hence the set of pairs of accessory parameters of HE sharing the same monodromy data. We complete the proof of Theorem 7 .

\section{Asymptotics of the accessory parameters}

In this section, we will derive some asymptotic approximations for the isomonodromy sets of accessory parameters of some Heun class equations expressed in terms of the monodromy data. The derivation are based on the connection between the accessory parameters and the the Laurent or Taylor coefficients for the corresponding Painlevé functions obtained in the previous sections and the asymptotic expansions for the Painlevé transcendents known in the literature. The equations of Heun class we considered in the section include the RBHE, CHE, and DHE. 


\subsection{Asymptotics of the accessory parameter of RBHE}

Consider the RBHE (1.7) with the accessory parameters $\left(a_{n}, q_{n}\right)$ such that the equation corresponding to these parameters has the monodromy data (4.9) specified by

$$
s_{-1}=-e^{-2 \alpha \pi i}, \quad s_{0}=e^{-2 \beta \pi i}, \quad s_{1}=-e^{2 \alpha \pi i},
$$

with $i \beta \in \mathbb{R}$ and $\alpha>-1 / 2$. The corresponding Painlevé XXXIV transcendents and their associated Lax pair play important roles in random matrix theory when a Fisher-Hartwig singularity located at an interior point where the density of the equilibrium density vanishes quadratically [4], or at the right edge of the spectrum where typically the density vanishes like a square root [18, 34]. The asymptotics of the Painlevé XXXIV transcendents and their associated $\tau$ functions have been worked out in [9, 18, 34]. Using these asympotics and the relation between the isomonodromy set of accessory parameters and the corresponding Painlevé XXXIV transcendents given in Theorem 3, we derive the asymptotic behavior of the accessory parameters $\left(a_{n}, q_{n}\right)$ expressed in terms of the given monodromy data (9.1).

Theorem 8. Let $\left(a_{n}, q_{n}\right)$ be the sequence of accessory parameters such that the RBHE (1.7) corresponding to these parameters has the same monodromy data (9.1), then we have the asymptotic approximations as $n \rightarrow \infty$

$$
\frac{4}{3}\left|a_{n}\right|^{\frac{3}{2}}=2 n \pi+2 i \beta \ln 3 n \pi+4 i \beta \ln 2-2 \arg \Gamma(\alpha-\beta)+\frac{1}{2}(2 \alpha+1) \pi+O\left(\frac{\ln n}{n}\right),
$$

and

$$
q\left(a_{n}\right)=2 \beta i\left|a_{n}\right|^{\frac{1}{2}}-\frac{1}{2}\left(\alpha-\alpha^{2}+3 \beta^{2}\right) a_{n}^{-1}+O\left(\left|a_{n}\right|^{-\frac{5}{2}}\right) .
$$

Proof. According to [34, Theorem 2] and [9, Proposition 3.4], we have the asymptotic behaviors for the Painlevé XXXIV transcendents $y(x)$ and the associated $\frac{\mathrm{d}}{\mathrm{d} x} \log \tau(x)$, corresponding to the Stokes multipliers (9.1), as $x \rightarrow-\infty$ :

$$
\begin{aligned}
y(x)= & \frac{2|\alpha-\beta|}{\sqrt{|x|}} \cos \left(\frac{\theta(x)}{2}+\arg \Gamma(1+\alpha-\beta)-\frac{\pi}{4}\right) \\
& \times \cos \left(\frac{\theta(x)}{2}+\arg \Gamma(\alpha-\beta)+\frac{\pi}{4}\right)+O\left(x^{-2}\right),
\end{aligned}
$$

and

$$
\begin{aligned}
\frac{\mathrm{d}}{\mathrm{d} x} \log \tau(x)= & 2 \beta i|x|^{\frac{1}{2}}-\frac{|\alpha-\beta|}{2 x} \sin (\theta(x)+2 \arg \Gamma(\alpha-\beta)+\arg (\alpha-\beta)) \\
& +\frac{\alpha^{2}-3 \beta^{2}}{2 x}+O\left(|x|^{-\frac{5}{2}}\right),
\end{aligned}
$$

where

and $\beta \in i \mathbb{R}$.

$$
\theta(x)=\frac{4}{3}|x|^{\frac{3}{2}}-3 i \beta \ln |x|-\alpha \pi-6 i \beta \ln 2,
$$

It follows from (9.4) that $y(x)$ admits a sequences of simple zeros lying on the negative real axis and tending to infinity with the asymptotic approximation given in (9.2). Moreover, the leading coefficient of the Taylor expansion of $y(x)$ near the zero $a_{n}$ is $2 \alpha$. The relation in the second equation of (4.20), together with (9.2) and (9.5), then implies (9.3). This completes the proof of Theorem 8 . 


\subsection{Asymptotics of the accessory parameter of $\mathrm{CHE}$}

In this subsection, we consider the CHE (7.25) with the accessory parameters $\left(a_{n}, q_{n}\right)$ such that the equation corresponding to these parameters has the monodromy data (7.6) parameterized in terms of $\sigma$ and $s$ as given in (7.9)-(7.10). In the seminal work 19, Jimbo derived the asymptotic expansion for the PV tanscendents and the associated $\tau$-function corresponding to the monodromy data. Using these asympotics and the relation between the isomonodromy set of accessory parameters and the corresponding Painlevé V transcendents given in Theorem 6, we derive the asymptotic behavior of the accessory parameters $\left(a_{n}, q_{n}\right)$ expressed in terms of the given monodromy data.

Theorem 9. Let $\left(a_{n}, q_{n}\right)$ be the sequence of accessory parameters such that the CHE (7.25) corresponding to these parameters has the same monodromy data (7.6) parameterized in terms of $\sigma$ and $s$ as given in (7.9) $-(7.10)$, then we have the asymptotic approximations as $n \rightarrow \infty$

$$
\ln \left|a_{n}\right| \sim\left(\operatorname{Re} \sigma \ln \left|c_{0}\right|+\operatorname{Im} \sigma \arg c_{0}-2 \pi n|\operatorname{Im} \sigma|\right) /|\sigma|^{2},
$$

and

$$
q\left(a_{n}\right)=\frac{\sigma^{2}-\left(\theta_{0}+\theta_{1}\right)^{2}}{4}-\left[\frac{\left(\theta_{\infty}-1\right)\left(\theta_{1}^{2}-\left(\theta_{0}-1\right)^{2}\right)}{4\left(\sigma^{2}-1\right)}+\frac{1-2 \theta_{0}-\theta_{\infty}}{4}\right] a_{n}+O\left(a_{n}^{2}\right),
$$

where $c_{0}=\frac{\left(\sigma+\theta_{\infty}\right)\left(\sigma+\theta_{0}+\theta_{1}\right) \Gamma(1+\sigma)^{2} \Gamma\left(\frac{1}{2}\left(\theta_{1}+\theta_{0}-\sigma\right)+1\right) \Gamma\left(\frac{1}{2}\left(\theta_{1}-\theta_{0}-\sigma\right)+1\right) \Gamma\left(\frac{1}{2}\left(\theta_{\infty}-\sigma\right)+1\right)}{\left(\sigma-\theta_{\infty}\right)\left(\sigma-\theta_{0}-\theta_{1}\right) \Gamma(1-\sigma)^{2} \Gamma\left(\frac{1}{2}\left(\theta_{1}+\theta_{0}+\sigma\right)+1\right) \Gamma\left(\frac{1}{2}\left(\theta_{1}-\theta_{0}+\sigma\right)+1\right) \Gamma\left(\frac{1}{2}\left(\theta_{\infty}+\sigma\right)+1\right)} \frac{1}{s}$ with $\operatorname{Im} \sigma \neq 0$ and $s \neq 0$.

Proof. According to [19, we have the following asymptotic expansion for the $\tau$-function of PV as $x \rightarrow 0$

$$
\begin{gathered}
\tau(x) \sim \text { const. } x^{\frac{1}{4}\left(\sigma^{2}-\theta_{\infty}^{2}\right)} \\
\quad \times\left\{1-\frac{\theta_{\infty}\left(\sigma^{2}+\theta_{1}^{2}-\theta_{0}^{2}\right)}{4 \sigma^{2}} x-\frac{\left(\sigma-\theta_{\infty}\right)\left[\left(\sigma-\theta_{1}\right)^{2}-\theta_{0}^{2}\right]}{8 \sigma^{2}(1+\sigma)^{2}} x\left(\rho x^{\sigma}\right)\right. \\
\left.+\frac{\left(\sigma+\theta_{\infty}\right)\left[\left(\sigma+\theta_{1}\right)^{2}-\theta_{0}^{2}\right]}{8 \sigma^{2}(1-\sigma)^{2}} x\left(\rho x^{\sigma}\right)^{-1}+\sum_{j=2}^{\infty} x^{j} \sum_{k=-j}^{j} c_{j k} x^{k \sigma}\right\}, \\
\rho=\frac{\Gamma(1-\sigma)^{2} \Gamma\left(\frac{1}{2}\left(\theta_{1}+\theta_{0}+\sigma\right)+1\right) \Gamma\left(\frac{1}{2}\left(\theta_{1}-\theta_{0}+\sigma\right)+1\right) \Gamma\left(\frac{1}{2}\left(\theta_{\infty}+\sigma\right)+1\right)}{\Gamma(1+\sigma)^{2} \Gamma\left(\frac{1}{2}\left(\theta_{1}+\theta_{0}-\sigma\right)+1\right) \Gamma\left(\frac{1}{2}\left(\theta_{1}-\theta_{0}-\sigma\right)+1\right) \Gamma\left(\frac{1}{2}\left(\theta_{\infty}-\sigma\right)+1\right)} s .
\end{gathered}
$$

Here the parameters $\sigma \neq 0$ and $s$ are the parameterization of the monodromy data of the corresponding CHE as given in (7.8)-(7.10).

As mentioned in [19, Remark 1], the asymptotics of the solution $y$ of PV can also be obtained from the asymptotic analysis carried out therein. The small- $x$ asymptotic expansion for $y$ was also derived in [27]. For $\operatorname{Im} \sigma \neq 0$, we see from the asymptotics of the PV tanscendents $y$ that it admits a sequence of simple poles $\left\{a_{n}\right\}_{n \in \mathbb{N}}$ such that

$$
a_{n}^{\sigma} \sim c_{0}, \quad c_{0}=\frac{\left(\sigma+\theta_{\infty}\right)\left(\sigma+\theta_{0}+\theta_{1}\right)}{\left(\sigma-\theta_{\infty}\right)\left(\sigma-\theta_{0}-\theta_{1}\right)} \rho^{-1}, \quad \text { as } \quad a_{n} \rightarrow 0 .
$$

The sequence of poles are clustering at zero along the spiral described by

$$
|\sigma|^{2} \ln \left|a_{n}\right|-\operatorname{Re} \sigma \ln \left|c_{0}\right|-\operatorname{Im} \sigma \arg c_{0} \sim-2 \pi n|\operatorname{Im} \sigma|,
$$


and

$$
|\sigma|^{2} \arg a_{n}-\operatorname{Re} \sigma \arg c_{0}+\operatorname{Im} \sigma \ln \left|c_{0}\right| \sim 2 \pi n \operatorname{Re} \sigma ;
$$

see for instance [27, Theorem 2.8]. It can be checked that the residues of $y$ at $a_{n}$ equal $\varepsilon_{+}$as given in (7.24).

Thus, from (9.9) and (9.11) we have (9.6). Substituting (9.8), (9.10) into (7.26), we obtain (9.7). This completes the proof of Theorem 9 .

It should be mentioned that similar formulae for the asymptotic approximations of the accessory parameters as $a \rightarrow 0$ have also been derived in [6] by considering the zeros of a special $\tau$-function of PV, with applications in black holes; see [6, (2.62a)-(2.62c)]. In general, the asymptotic expansions of the PV solutions $y(x)$ and the associated $\tau$-functions near infinity are rather complicated. There exists no general asymptotic expansions for $y(x)$ or $\tau(x)$ as $x \rightarrow \infty$ except along some special rays: $\arg x=0, \pi / 2, \pi, 3 \pi / 2$; see [2, 21, 28]. In [2, 28, the asymptotics for $y(x)$ and the logarithmic derivative of the $\tau$-functions as $x \rightarrow i \infty$ are established. From the asymptotic expansions, it is shown in [28] that under certain conditions the PV solutions admit sequences of poles and of zeros lying on the imaginary axis and tending to $i \infty$. Combining these results with the expressions of the accessory parameters of CHE in terms of the $\tau$-function given in (7.26)-(7.27), the asymptotic approximations of the isomonodromic set of accessory parameters $\left(a_{n}, q_{n}\right)$ corresponding to some special monodromy data may also be obtained.

\subsection{Asymptotics of the accessory parameter of DHE}

In the pioneering work of McCoy, Tarcy and $\mathrm{Wu}$ [24], the asymptotics and the connection formulae for one-parameter family of solutions to PIII were derived rigorously. These solutions have important applications in the analysis of two-dimensional Ising model [33. More precisely, they showed in [24] that there are one-parameter family of solutions of PIII with the asymptotics

$$
y(x ; \nu, \lambda) \sim 1-\lambda \Gamma\left(\nu+\frac{1}{2}\right) 2^{-2 \nu} x^{-\nu-\frac{1}{2}} e^{-2 x}\left(1+\sum_{j=1}^{\infty} \frac{c_{j}}{x^{j}}\right), \quad x \rightarrow+\infty,
$$

where parameters $\theta_{0}, \theta_{\infty}$ satisfy the relation

$$
\theta_{0}=\theta_{\infty}-1=\nu .
$$

For $|\lambda|<1 / \pi$, the asymptotic behavior of $y(x ; \nu, \lambda)$ as $x \rightarrow 0^{+}$is described by

$$
y(x ; \nu, \lambda) \sim B(2 x)^{\sigma}
$$

with the parameters $\sigma$ and $B$ are given as explicit functions of $\lambda$ and $\nu$, which are now known as the connection formulae. When $\lambda>1 / \pi$ and the parameter $\nu=0$, the asymptotic behavior of $y(x)$ as $x \rightarrow 0^{+}$was also derived in [24]

$$
y(x ; 0, \lambda)=\frac{x}{2 \mu} \sin \left\{2 \mu \ln \frac{x}{4}-2 \arg \Gamma(i \mu)\right\}+O\left(x^{3}\right), \quad x \rightarrow 0^{+},
$$

where

$$
\lambda=\frac{1}{\pi} \cosh (\pi \mu), \quad \mu>0 .
$$

For $\lambda<-1 / \pi$, the asymptotics of $y(x)$ as $x \rightarrow 0^{+}$follows from (9.14) and the symmetry relation [24, (4.127)]

$$
y(x ; \nu, \lambda)=\frac{1}{y(x ; \nu,-\lambda)} .
$$


The asymptotic formula for general parameter $\nu \in \mathbb{R}$ was worked out in [11]. When $\lambda>1 / \pi$ and $\nu=0$, it is readily seen from (9.14) that $y(x ; 0, \lambda)$ admits a sequence of zeros $\left\{c_{n}\right\}_{n \in \mathbb{N}}$ lying on the positive real axis with $x=0$ being a limiting point:

$$
c_{n} \sim 4 \exp \left\{-\frac{n \pi}{\mu}+\frac{\arg \Gamma(i \mu)}{\mu}\right\} \rightarrow 0^{+}, \quad n \rightarrow \infty .
$$

Moreover, it is straightforward to check that the Taylor expansions of $y$ at the zeros $\left\{c_{2 n}\right\}_{n \in \mathbb{N}}$ and $\left\{c_{2 n+1}\right\}_{n \in \mathbb{N}}$ are corresponding to $\sigma=\sigma_{+}$and $\sigma=\sigma_{-}$in (5.29), respectively. It is also seen from the relation (9.15) that there are infinitely many poles of $y(x ; 0, \lambda)$ clustering at $x=0$ on the positive real axis when $\lambda<-1 / \pi$.

Let us consider the DHE equation (1.3) with the accessory parameters $a$ and $q$ and the fixed parameters $\gamma=1+\theta_{0}$ and $p=\frac{1}{4}\left(\theta_{\infty}+\theta_{0}\right)$. Applying Theorem 4, there is an isomonodromy set of pairs of accessory parameters $\left(a_{n}, q_{n}\right)$ such that the DHE equation corresponding to these parameters have the same monodromy data as the Painlevé III transcendent $y(x ; 0, \lambda)$ determined by the asymptotic behavior (9.14). Moreover, the parameter $a_{n}=c_{2 n}$ and the accessory parameters $q_{n}$ are expressed in terms of the Laurent parameters of the PIII transcendents as given in (5.31). Combining (5.16), (5.31), (9.14) and (9.16), we obtain the asymptotic approximation of the accessory parameters as stated in the following theorem.

Theorem 10. Let $\left(a_{n}, q_{n}\right)$ be the sequence of accessory parameters such that the DHE equation (1.3) corresponding to these parameters has the same monodromy data as the Painlevé III transcendents $y(x ; 0, \lambda)$ determined by the asymptotic behavior (9.14), then we have the asymptotic approximations as $n \rightarrow \infty$

$$
a_{n} \sim 4 \exp \left\{-\frac{2 n \pi}{\mu}+\frac{\arg \Gamma(i \mu)}{\mu}\right\} \rightarrow 0^{+},
$$

and

$$
q\left(a_{n}\right)=-\frac{\mu^{2}+1}{4}+O\left(a_{n}^{2}\right) .
$$

Here the parameter $\lambda=\frac{1}{\pi} \cosh (\pi \mu)$ and $\mu>0$.

Remark 11. In Theorem 10, we have derived the asymptotics of a isomonodromy sequence of accessory parameters for DHE (1.3). The asymptotics are expressed in terms of the parameters in the behavior (9.14) of the corresponding Painlevé III transcendents. It would be desirable to describe the asymptotics via the monodomy data as given in Theorem 8 and Theorem 9 . However, to the best of our knowledge the connection between the parameters in the asymptotic behavior (9.14) for the Painlevé III transcendents and the monodomy data has not been worked out in the literature. We expect that such a connection formula could be derived, perhaps by using the Riemann-Hilbert method or Isomonodromy method [12. This, together with Theorem 10. would then give us a description of the asymptotics of the accessory parameters for DHE via the monodomy data. We will leave this problem to a future consideration.

\section{Acknowledgements}

The authors are very grateful to the anonymous reviewers for their constructive comments and suggestions. The work of Shuai-Xia Xu was supported in part by the National Natural Science Foundation of China under grant numbers 11571376 and 11971492. Yu-Qiu Zhao was supported in part by the National Natural Science Foundation of China under grant numbers 11571375 and 11971489 . 


\section{References}

[1] J.B. Amado, B.C. da Cunha and E. Pallante, On the Kerr-AdS/CFT correspondence, J. High Energy Phys., 2017 (2017), 08(2017)094.

[2] F.V. Andreev and A.V. Kitaev, Connection formulae for asymptotics of the fifth Painlevé transcendent on the imaginary axis: I, Stud. Appl. Math., 145 (2020), 397-482.

[3] T. Anselmo, R. Nelson, B.C. da Cunha and D.G. Crowdy, Accessory parameters in conformal mapping: exploiting the isomonodromic tau function for Painlevé VI, Proc. Roy. Soc. Lond. A, 474 (2018), 20180080.

[4] T. Claeys, A.B.J. Kuijlaars and M. Vanlessen, Multi-critical unitary random matrix ensembles and the general Painlevé II equation, Ann. Math., 168 (2008), 601-641.

[5] P.A. Clarkson, Painlevé equations-nonlinear special functions, Orthogonal polynomials and special functions, 331-411, Lecture Notes in Math., 1883, Springer, Berlin, 2006.

[6] B.C. da Cunha and J.P. Cavalcante, Confluent conformal blocks and the Teukolsky master equation, to appear in Phys. Rev. D, arXiv:1906.10638.

[7] B.C. da Cunha and F. Novaes, Kerr scattering coefficients via isomonodromy, J. High Energy Phys., 2015 (2015), 11(2015)144.

[8] B.C. da Cunha and F. Novaes, Kerr-de Sitter greybody factors via isomonodromy, Phys. Rev. D, 93 (2016), 024045.

[9] D. Dai, S.-X. Xu and L. Zhang, On integrals of the tronquée solutions and the associated Hamiltonians for the Painlevé II equation, J. Differential Equations, 269 (2020), 24302476 .

[10] B. Dubrovin and A.A. Kapaev, A Riemann-Hilbert approach to the Heun equation, SIGMA, 14 (2018), 093.

[11] M. Fasondini, B. Fornberg and J.A.C. Weideman, A computational exploration of the McCoy-Tracy-Wu solutions of the third Painlevé equation, Phys. D, 363 (2018), 18-43.

[12] A.S. Fokas, A.R. Its, A.A. Kapaev and V.Y. Novokshenov, Painlevé transcendents: The Riemann-Hilbert approach, Math. Surv. Monog., Vol. 128, Amer. Math. Soc., Providence, RI, 2006.

[13] P.J. Forrester, Log-gases and random matrices, London Mathematical Society Monographs Series, 34., Princeton University Press, Princeton, NJ, 2010.

[14] R. Fuchs, Sur quelques équations différentielles linéaires du second ordre, C. R. Acad. Sci. Paris, 141 (1906), 555-558.

[15] R. Fuchs, Über lineare homogene Differentialgleichungen zweiter Ordnung mit drei im Endlichen gelegenen wesentlich singulären Stellen, Math. Ann., 63 (1907), 301-321

[16] V.I. Gromak, I. Laine and S. Shimomura, Painlevé differential equations in the complex plane, de Gruyter Studies in Mathematics, Vol. 28, Walter de Gruyter GmbH \& Co. KG, Berlin, 2002. 
[17] M. Hortaçsu, Heun functions and their uses in physics, in Proceedings of the 13th Regional Conference on Mathematical Physics, Editors U. Camci, I. Semi, World Sci. Publ., Hackensack, NJ, 2013, 23-39.

[18] A.R. Its, A.B.J. Kuijlaars, J.Östensson, Critical edge behavior in unitary random matrix ensembles and the thirty fourth Painlevé transcendent, Int. Math. Res. Not., 2008 (2008), rnn017.

[19] M. Jimbo, Monodromy problem and the boundary condition for some Painlevé equations, Publ. Res. Inst. Math. Sci., 18 (1982), 1137-1161.

[20] M. Jimbo and T. Miwa, Monodromy preserving deformation of linear ordinary differential equations with rational coefficients. II, Phys. D, 2 (1981), 407-448.

[21] O. Lisovyy, H. Nagoya and J. Roussillon, Irregular conformal blocks and connection formulae for Painlevé V functions, J. Math. Phys., 59 (2018), 091409.

[22] A. Litvinov, S. Lukyanov, N. Nekrasov and A. Zamolodchikov, Classical conformal blocks and Painlevé VI, J. High Energy Phys., 2014 (2014), 07(2014)144.

[23] D. Masoero and P. Roffelsen, Poles of Painlevé IV rationals and their distribution, SIGMA, 14 (2018), 002.

[24] B.M. McCoy, C.A. Tracy and T.T. Wu, Painlevé functions of the third kind, J. Math. Phys., 18 (1977) 1058-1092.

[25] F.W.J. Olver, D.W. Lozier, R.F. Boisvert and C.W. Clark (Eds.), NIST handbook of mathematical functions, Cambridge University Press, New York, 2010.

[26] A. Ronveaux (Ed.), Heun's differential equations, Oxford University Press, Oxford-New York-Tokyo, 1995.

[27] S. Shimomura, Critical behaviours of the fifth Painlevé transcendents and the monodromy data, Kyushu J. Math., 71 (2017), 139-185.

[28] S. Shimomura, Three-parameter solutions of the PV Schlesinger-type equation near the point at infinity and the monodromy data, SIGMA, 14 (2018), 113.

[29] S. Yu. Slavyanov, Painlevé equations as classical analogues of Heun equations, J. Phys. A, 29 (1996), 7329-7335.

[30] S. Yu. Slavyanov, Isomonodromic deformations of Heun and Painlevé equations, Theor. Math. Phys., 123 (2000),744-753.

[31] S. Yu. Slavyanov and W. Lay, Special functions: a unified theory based on singularities, Oxford Mathematical Monographs, Oxford University Press, Oxford, 2000.

[32] S. Yu. Slavyanov, W. Lay and A. Seeger, Classification of Heun's differential equations, Oxford University Press, Oxford, 1995.

[33] T.T. Wu, B.M. McCoy, C.A. Tracy and E. Barouch, Spin-spin correlation functions for the two-dimensional Ising model: exact theory in the scaling region, Phys. Rev. B, 13 (1976), $316-374$.

[34] X.-B. Wu, S.-X. Xu and Y.-Q. Zhao, Gaussian unitary ensemble with boundary spectrum singularity and $\sigma$-form of Painlevé II, Stud. Appl. Math., 140 (2018), 221-251. 